Document downloaded from:

http://hdl.handle.net/10251/121396

This paper must be cited as:

Ossorio-García, J.; Melgarejo-Lermas, JC.; Boria Esbert, VE.; Guglielmi, M.; Bandler, J. (2018). On the Alignment of Low-Fidelity and High-Fidelity Simulation Spaces for the Design of Microwave Waveguide Filters. IEEE Transactions on Microwave Theory and Techniques. 66(12):5183-5196. https://doi.org/10.1109/TMTT.2018.2871022

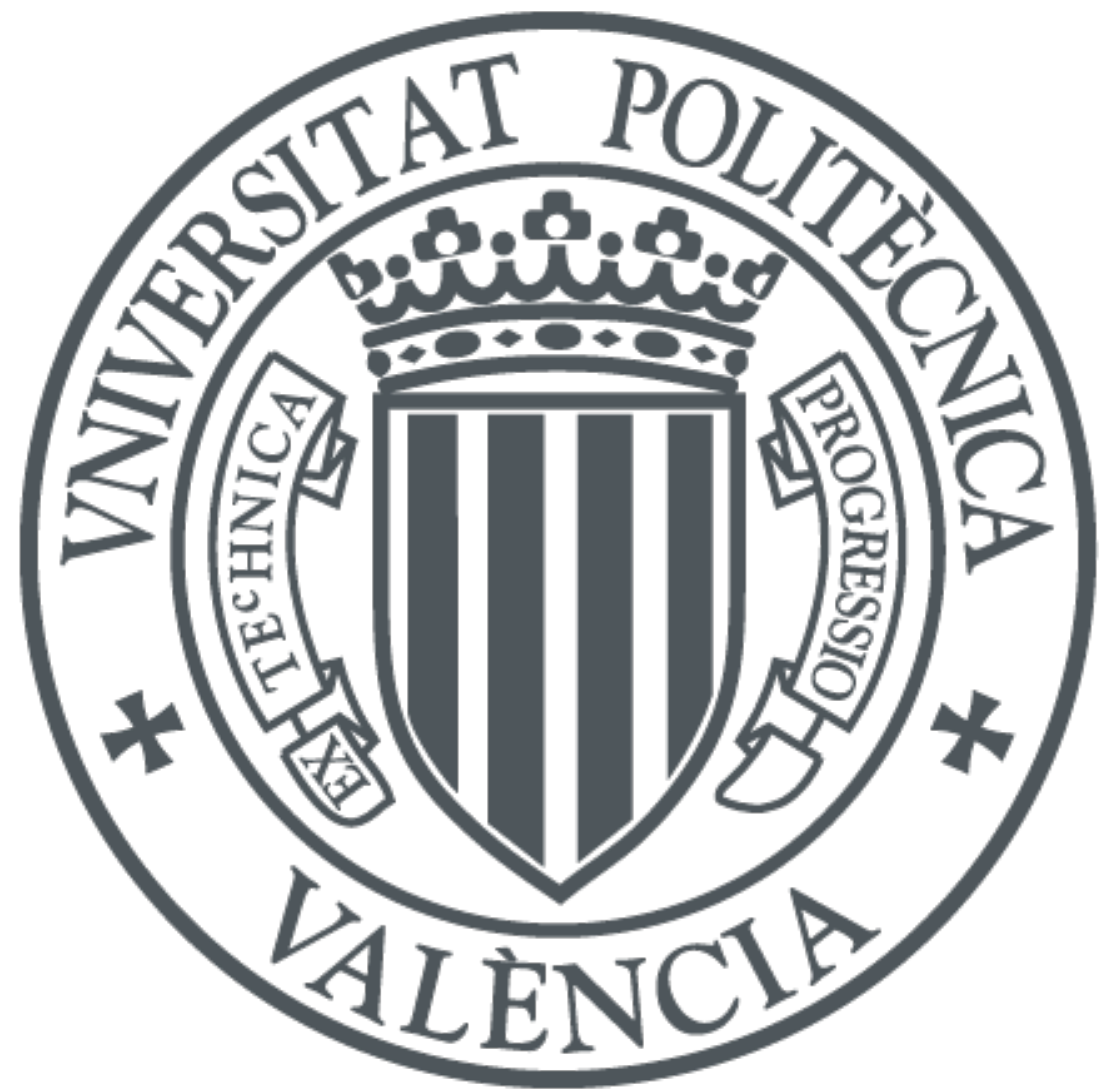

The final publication is available at

http://doi.org/10.1109/TMTT.2018.2871022

Copyright Institute of Electrical and Electronics Engineers

Additional Information 


\title{
On the Alignment of Low-Fidelity and High-Fidelity Simulation Spaces for the Design of Microwave Waveguide Filters
}

\author{
Javier Ossorio, Juan C. Melgarejo, Vicente E. Boria, Fellow, IEEE, Marco Guglielmi, Fellow, IEEE, and John W. \\ Bandler, Life Fellow, IEEE
}

\begin{abstract}
The objective of this paper is to advance the stateof-the-art of Aggressive Space Mapping (ASM) by demonstrating how, for resonant structures, and microwave filters in particular, an ASM based optimization procedure may converge to the desired target performance in just one step. This behavior is first justified in physical terms, using the Electromagnetic (EM) cavity perturbation theory, and is then investigated numerically. Several practical filter design demonstrations are also presented. The paper is concluded with a conceptual definition of Space Alignment in the context of ASM.
\end{abstract}

Index Terms-Aggressive Space Mapping (ASM), optimization, microwave filter, engineering design, spaces, waveguide filters.

\section{INTRODUCTION}

$\mathbf{S}$ PACE MAPPING (SM) was first introduced by Prof. John W. Bandler in 1994 [1]. SM is essentially an optimization procedure that is based on the use of two different simulation algorithms (or spaces) that describe the same object (a microwave component for instance). In particular, the first is a fast but not accurate simulation algorithm (the coarse or low fidelity model), while the second is a highly accurate but computationally expensive algorithm (the fine or high fidelity model). The second model could also simply be the measured electrical behavior of the component. The basic idea behind $\mathrm{SM}$ is that, under certain conditions, it is possible to extract useful information about the behavior of the fine model by performing computations with the coarse model only. As the name indicates, the basic objective of SM is to establish a mapping between the coarse and fine models. The correct mapping is usually obtained with an iterative procedure, where the number of steps required is linked to the number of variables that need to be optimized. A further development of this approach is the Aggressive Space Mapping (ASM) [2].

In the ASM approach (see details in [2]), the number of initial fine simulations required in the original implementation of the SM algorithm [1] are reduced by first using the

Manuscript received Month DD, YYYY; revised Month DD, YYYY; accepted Month DD, YYYY.

This work has been supported by MINECO (Spanish Government) under R\&D project TEC2016-75934-C4-1-R and R\&D Technicians grant PTA201510839-I.

J. Ossorio, J. C. Melgarejo, V. E. Boria and M. Guglielmi are with Departamento de Comunicaciones, iTEAM, Universitat Politècnica de València, E-46022, Spain (e-mails: jaosgar@teleco.upv.es, juamelle@teleco.upv.es, vboria@dcom.upv.es, marco.guglielmi@iteam.upv.es).

J. W. Bandler is with the Department of Electrical and Computer Engineering, McMaster University, Hamilton, ON L8S 4K1, Canada, and with Bandler Corporation, Dundas, ON L9H 5E7, Canada (email: bandler@mcmaster.ca). identity matrix as the initial space mapping matrix. Then, the ASM method employs a quasi-Newton iteration together with the update of the space mapping matrix by means of the classic Broyden formula [3]. Due to its simplicity and ease of implementation, this very simple ASM concept evolved, over the years, into an extremely powerful and widely used family of optimization procedures [4]. A very large number of contributions concerning ASM can indeed be found in the technical literature in very different areas of investigation [5]. In particular, initial ASM implementations for the design of microwave filters appear in [6] and [7], where ASM is used to design inductive microwave filters with rounded corners. In addition, ASM techniques have also been used for the design of multiplexers based on dielectric-resonator filters [8], where the number of ASM iterations needed are high due to the numbers of variables involved in the complete design process.

Furthermore, several contributions have been recently published with the aim of reducing the number of ASM iterations (and the related cost) by focusing the efforts on improving the parameter extraction optimization algorithms ([9] to [11]), on refining the fine models employed (even using measured data) [12], [13], and a variety of other techniques ([14] to [17]). The practical usage of all these advances with different types of microwave filters can be found in [18].

Finally, a very recent contribution, aimed at the efficient design and implementation of the ASM technique [19], showed that it is possible to design an inductive rectangular waveguide filter with ASM in just one step, using as coarse model a Multimode Equivalent Network (MEN) representation of the filter structure [20]. This result, however, is demonstrated in [19] without a proper theoretical explanation.

In this context, therefore, the objective of this paper is to fill this gap by showing how and why, for resonant structures in general, and for waveguide filters in particular, an ASM based design and optimization procedure may converge to the desired target performance in just one step, independently of the number of variables involved in the optimization process.

This remarkable behavior is first justified in terms of the well known EM cavity perturbation theory, and is then fully demonstrated numerically with a basic resonant structure. In addition to theory, a number of practical filter design examples are designed, indicating under what conditions an ASM based design and optimization procedure may (or may not) converge in just one step. The paper is concluded with a definition of space alignment in the context of ASM. 


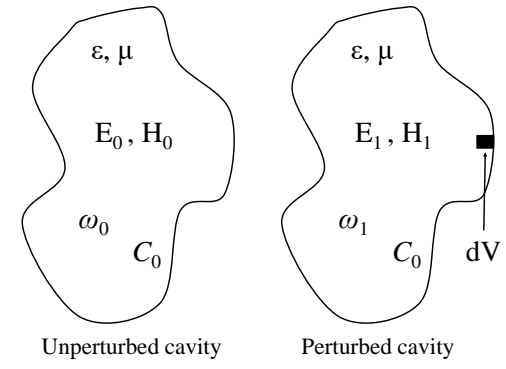

Fig. 1. Unperturbed and perturbed cavities.

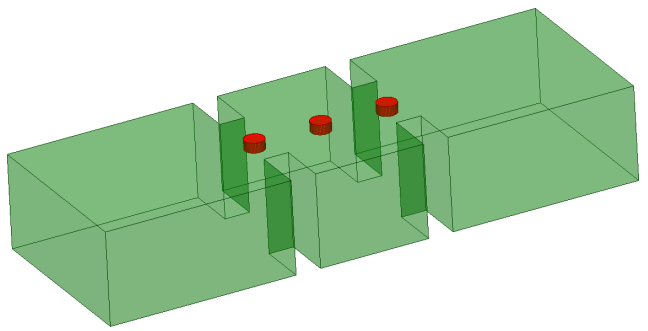

Fig. 2. One-pole tunable filter structure.

\section{CAVity Perturbation Theory}

The basic behavior of a microwave cavity perturbed by a small shape variation has been known for quite some time [21]. The basic effect that can be observed, by introducing a small perturbation $d V$ in the shape of a cavity (see Fig. 1), is a small shift in resonant frequency $\omega_{1}-\omega_{0}$ approximately given by:

$$
\frac{\omega_{1}-\omega_{0}}{\omega_{0}} \approx \frac{\Delta W_{m}-\Delta W_{e}}{W_{m}+W_{e}}
$$

where $W_{m}$ and $W_{e}$ represent the actual magnetic and electric energies contained in the cavity, and $\Delta W_{m}$ and $\Delta W_{e}$ represent the time-average magnetic and electric energies contained in $d V$.

Waveguide filters are usually composed of a sequence of resonant cavities coupled to each other by apertures. Furthermore, tuning elements (screws) are usually introduced both in the apertures and in the cavities in order to compensate for manufacturing errors. In this context, therefore, microwave filters can indeed be viewed as complex microwave cavities and, therefore, their behavior must follow the cavity perturbation theory.

\section{EXPERIMENTAL INVESTIGATION}

To explore this point in more detail, we now perform two simple experiments. Let us consider a filter composed of a single cavity as shown in Figs. 2 and 3, for the structure and the simulated performance, respectively. This is indeed the simplest possible filter. It is interesting to note now that the performance shown in Fig. 3 has been obtained with the full-wave electromagnetic (EM) simulator FEST3D from AuroraSat. One particular aspect of FEST3D is that the user can choose the level of accuracy with which the calculations are performed.

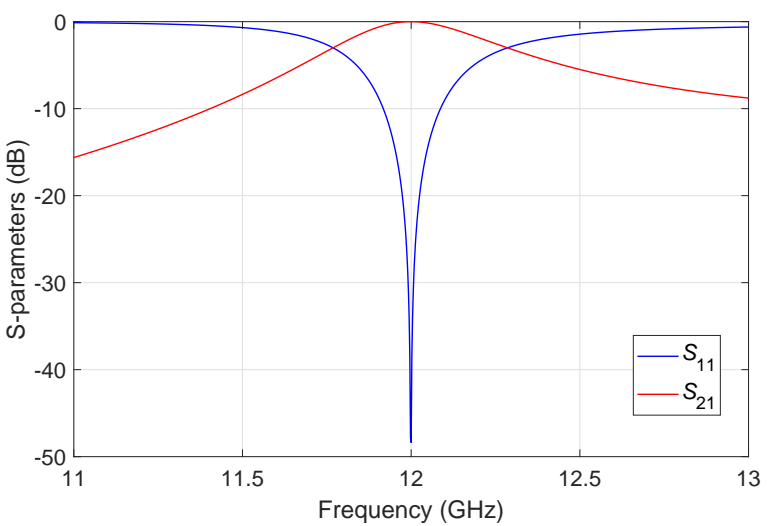

Fig. 3. One-pole tunable filter performance.

The computational parameters that affect the accuracy are:

- Accessible modes

- Number of basis functions

- Green's function terms

- Maximum frequency in the tuning cavities

Furthermore, one additional important point is that in the calculations performed by FEST3D, the geometrical details of the structure are always taken into account rigorously. What changes is the accuracy with which the geometry is represented in terms of the actual capacitance and inductance values in the multimode equivalent network produced. It is important to recall at this point that FEST3D is based on Multi-mode Equivalent Networks (MEN), which are effectively equivalent circuit representations of the structure being analyzed.

A complete and detailed description of the meaning and effect of the computational parameter values can be found in [20] (see page 232). In this context, the numerical values used in this paper are as follows:

- Accessible modes $=10$

- Number of basis functions $=30$

- Green's function terms $=300$

- Maximum frequency in the tuning cavities $=50 \mathrm{GHz}$

With this set of parameters, FEST3D is extremely fast computationally, however, it does not provide the maximum accuracy in terms of the electromagnetic performance of the structure in Fig. 2. We will, therefore, call this the Low Fidelity (LF) simulation.

We now perform another experiment, namely, we change the values of the computational parameters in FEST3D to the following values:

- Accessible modes $=100$

- Number of basis functions $=300$

- Green's function terms $=3000$

- Maximum frequency in the tuning cavities $=100 \mathrm{GHz}$

With this set of parameters, the accuracy of the EM computations is greatly increased.

This will be our high fidelity simulation (HF). Furthermore, the structure has been optimized so that the performance is exactly identical to the one shown in Fig. 3. Fig. 4 shows the two superimposed curves. 


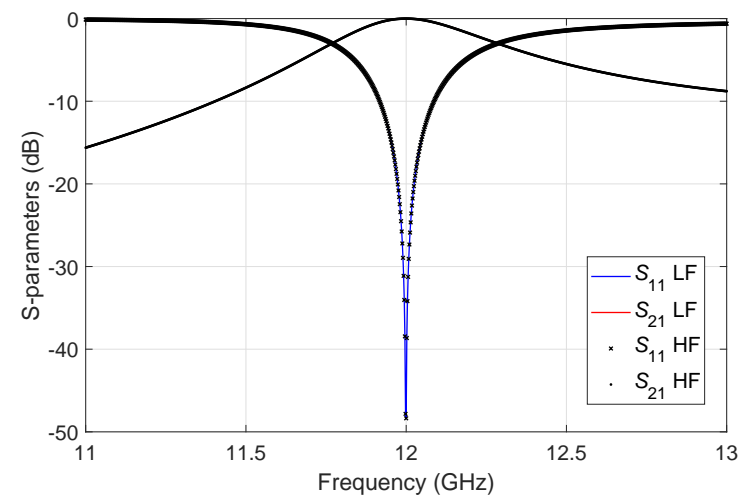

Fig. 4. High-Fidelity and Low-Fidelity one-pole tunable filter responses.

TABLE I

DIMENSIONS OF THE ONE-POLE STRUCTURE IN MILLIMETERS.

\begin{tabular}{|c|c|c|c|}
\hline Structure & Height & Width & Length \\
\hline I/O aperture & 9.525 & 9.488 & 3 \\
\hline Cavity & 9.525 & 19.05 & 11.921 \\
\hline
\end{tabular}

TABLE II

TUNING SCREW PENETRATIONS IN MILLIMETERS.

\begin{tabular}{|c|c|c|}
\hline Structure & Apertures screw & Cavity screw \\
\hline LF & 1.0 & 1.0 \\
\hline HF & 0.9108 & 0.9321 \\
\hline
\end{tabular}

As we can see, the two curves are practically coincident.

Naturally, however, in order to have the same exact response with two different simulations, something must be different in the structural dimensions. Indeed, the value of the penetration of the tuning screws both in the cavity and in the coupling apertures are different. As we can see in Table II, there is a small, but significant, difference in screw penetration.

We now have two different structures, or better, models or equivalent circuits, that give the same electrical response. It is important to note at this point that, although the two physical structures are slightly different, their respective equivalent circuits must contain the exact same amount of stored electric and magnetic energy. This is, in fact, the direct consequence of the fact that their electrical responses are identical.

Continuing with our experiments, we now make the observation that, if we consider the two simulations as two different objects, they must both obey the perturbation theory recalled in the previous section. As a consequence, if we change the screw penetration of the same amount in the two cases, we should obtain the exact same shift in frequency. Fig. 5 shows the result obtained by increasing the screw penetration in the cavity by $0.2 \mathrm{~mm}$ in both cases. As we can clearly see, once again we obtain two basically identical responses.

To further understand the behavior of this simple one pole filter, we now turn our attention to the tuning screws in the coupling apertures.

The following question comes naturally: do we obtain the same behavior if we change the penetration of the tuning screws in one of the apertures?

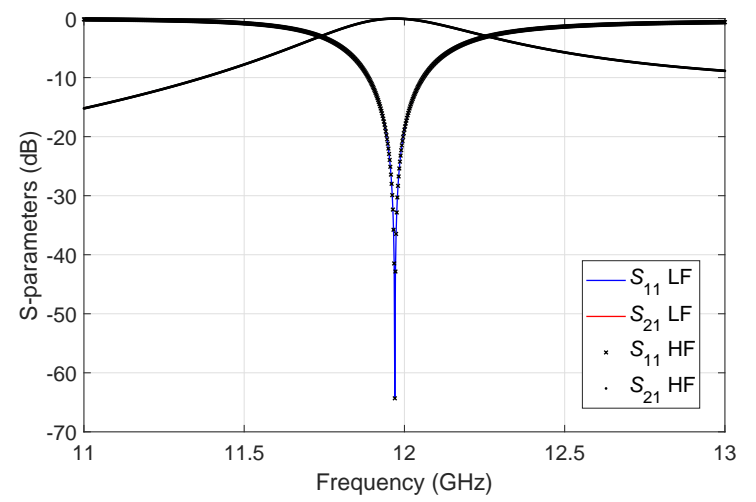

Fig. 5. High-Fidelity and Low-Fidelity one-pole detuned cavity filter responses.

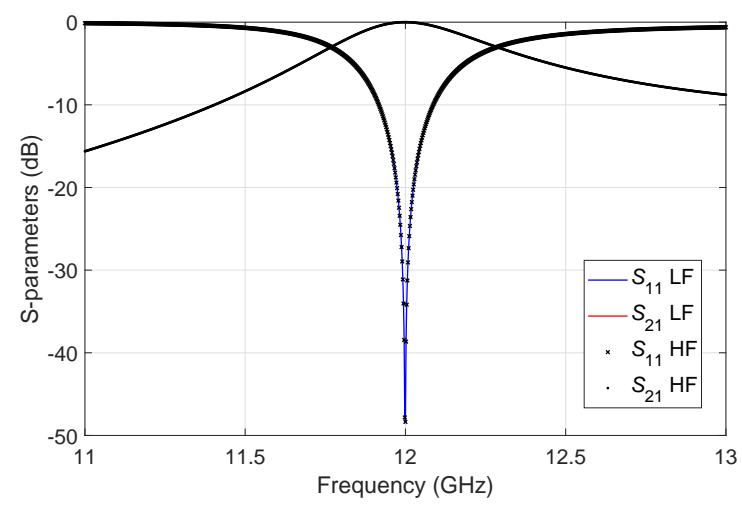

Fig. 6. High-Fidelity and Low-Fidelity one-pole detuned aperture filter responses.

Fig. 6 shows the results obtained with both simulation instances with the tuning screw in the one of the apertures penetrating $0.2 \mathrm{~mm}$ more.

As we can clearly see, we obtain once again practically coincident results.

To explore more in depth the behavior of the one-pole structure in Fig. 3 we now compare the numerical results of the HF and LF simulations in the vicinity of the resonance.

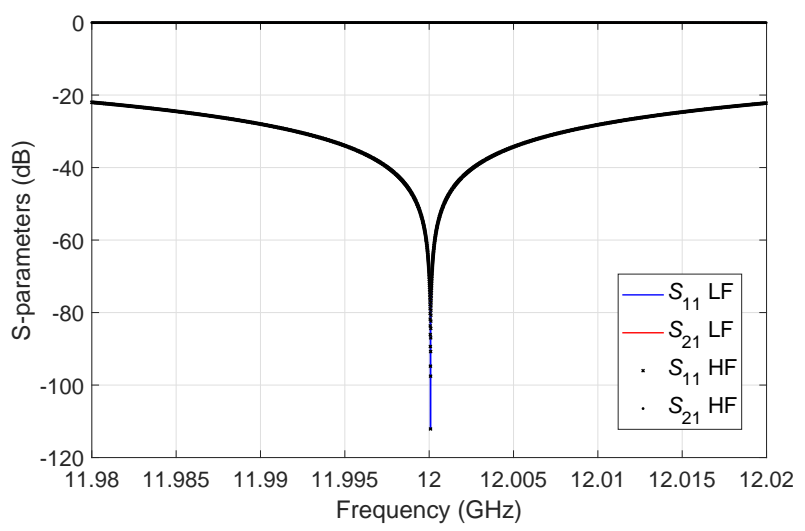

Fig. 7. High-Fidelity and Low-Fidelity one-pole filter simulations near resonance. 


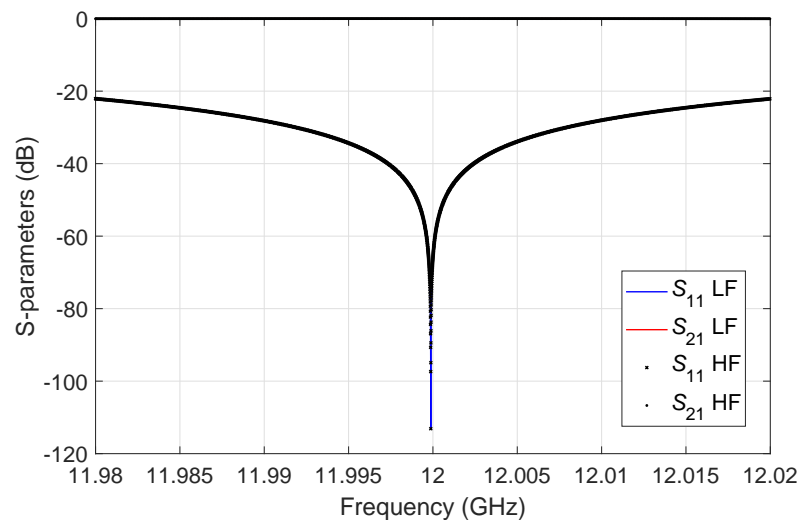

Fig. 8. High-Fidelity and Low-Fidelity simulations with detuned cavity near resonance.

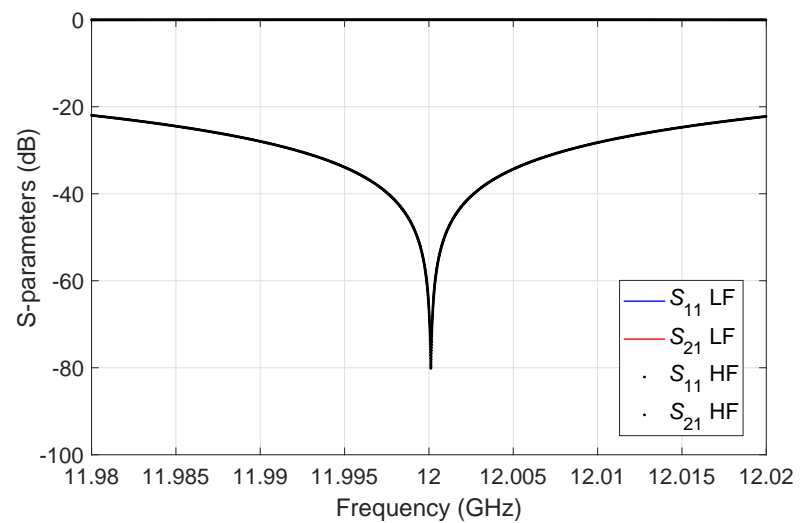

Fig. 9. High-Fidelity and Low-Fidelity simulations with detuned aperture near resonance.

As we can see from Fig. 7 , the two resonance frequencies are indeed identical, within the numerical accuracy of the simulations.

We now repeat the same comparison but with the cavities detuned by just one micron. As we can see from Fig. 8, also in this case the agreement between the detuned LF and HF simulations is virtually perfect (within the accuracy of the simulations).

The next test that we perform is with respect to the tuning screw in one of the coupling apertures. Fig. 9 shows the results obtained introducing again a difference of 1 micron. As we can see, once again, the agreement is virtually perfect. In next section, we will make use of these results for the numerical evaluation of the derivatives of both simulation spaces with respect to the geometrical parameters of the cavity.

\section{Detailed Numerical Investigation}

In order to further study and understand the behavior shown so far, we now look at the values of the numerical derivatives of the scattering parameters with respect to the tuning screw penetrations, in both the LF and HF simulation spaces. The computation of the numerical derivatives is in fact one of the key steps in any optimization procedure.
TABLE III

DIFFERENCE BETWEEN DERIVATIVES OF HF AND LF SIMULATIONS.

\begin{tabular}{|c|c|c|c|}
\hline Detuned & Av. deriv. vals. (LF-HF) & Av. err. & Err. (\%) \\
\hline$d\left|S_{11}\right| / d x$ Cav. & $0.5872-0.5905$ & 0.0033 & 0.559 \\
\hline$d\left|S_{11}\right| / d x$ Ap. & $0.1621-0.1637$ & 0.0016 & 0.9876 \\
\hline$d\left|S_{21}\right| / d x$ Cav. & $1.8312 e^{-4}-1.8412 e^{-4}$ & $1.002 e^{-6}$ & 0.547 \\
\hline$d\left|S_{21}\right| / d x$ Ap. & $4.8906 e^{-5}-4.8939 e^{-5}$ & $3.233 e^{-8}$ & 0.0661 \\
\hline
\end{tabular}

In this context, therefore, we will use the classic formula to compute the value of the numerical derivative at each frequency point in our simulation range, namely:

$$
f^{\prime}\left(x_{0}\right) \approx \frac{f\left(x_{0}+h\right)-f\left(x_{0}\right)}{h}
$$

where $f^{\prime}\left(x_{0}\right)$ is the derivative value, $h$ is the step (that is equal to the difference in screw penetration), and $f\left(x_{0}\right)$ and $f\left(x_{0}+h\right)$ represent the previous value of the function, and the new value of the function after the step, respectively. For the step $h$, we will use, as before, the value of one micron.

We are now going to compute the numerical derivatives (of the scattering parameters) in both the LF and in the HF spaces, and compute the difference. If the difference is zero (or better vanishingly small) than the two derivatives can be considered to be identical. The (average) difference is computed using the following standard expression:

$$
A v . E r r .=\sum_{i=1}^{N} \frac{\left|f^{\prime}\left(x_{0}\right)_{L F}-f^{\prime}\left(x_{0}\right)_{H F}\right|}{N}
$$

where $N$ is the number of frequency points where the calculations have been performed, and $f^{\prime}\left(x_{0}\right)_{L F}$ and $f^{\prime}\left(x_{0}\right)_{H F}$ are the values of the derivatives for the LF and HF spaces, respectively. Table III shows the (average) values obtained for the derivative of the scattering parameters (in $\mathrm{dB}$ ) with respect to the screw penetrations ( $d x=h$ in equation (2)), and the value of the errors, for a set of points near the resonant frequency (1dB S21 variation on both sides), for both the S21 and the S11 parameters. As we can see, the values of the errors are at least two order of magnitude smaller than the values of the derivatives. We have also verified that the error becomes even smaller, if the value of the step is decreased.

We can therefore conclude that the difference in the values of the derivatives is indeed vanishingly small (as $h \rightarrow 0$ ), and therefore, the derivatives themselves are essentially identical.

It is now interesting to note that, according to equations (5) and (6) of [4], the fact that the derivatives (or Jacobian) in the LF and HF spaces are identical, implies that the socalled Broyden matrix $B$ is, in fact, always equal to the identity matrix. This simple observation has, indeed, a very important consequence in the context of ASM techniques. It indicates that the ASM optimization approach for resonant structures, (and microwave filters in particular) may converge to the desired goal with only one step [19].

\section{One Step Aggressive Space Mapping}

Since the One Step Aggressive Space Mapping (OS-ASM) concept is the central topic of this paper, we will now explain in more detail what we mean with One Step. 
The first step in the conventional ASM design of microwave filters is to obtain a structure in the coarse (or low-fidelity) domain that satisfies the given set of requirements that is our design target. What we obtain at the end of this process is a set of numerical values for the physical parameters that define the performance of the microwave filter (for instance, a set of screw penetration values $X_{c}^{t}$ ).

The next step is the simulation of the exact same structure in the fine (or high fidelity) domain. Naturally, the result that we obtain in the fine domain is a detuned performance $\left(X_{c}^{t}=\right.$ $\left.X_{f}^{d t}\right)$. That is, the filter structure designed in the coarse domain does not satisfy the given requirements if simulated in the fine domain.

The next step is to recover with one optimization in the coarse domain the detuned performance obtained in the fine domain. What we obtain at the end of this step is another set of structural parameters that represents, in the coarse domain, the detuned performance obtained in the fine domain $\left(X_{c}^{d t}\right)$. We now have two sets of structural parameters in the coarse domain, and we can compute the distance between the two sets of parameters. This distance is, in fact, the difference between the sets of numerical values.

In practice, therefore, what we mean with OS-ASM is that if we add this difference in the inverse direction to the initial set of structural parameters in the fine domain, we obtain directly the desired optimal response in the fine domain.

In other words, from the initial coarse design, we need only one (optimization) step to obtain the desired final response in the fine domain.

It is interesting to note that what we described verbally in the previous paragraph can also be stated in mathematical terms. To do that, we first recall that a linear mapping between $X_{c}$ (coarse domain) and $X_{f}$ (fine domain) can be written in the form [5]:

$$
X_{c}=B * X_{f}+C
$$

where $B$ is the Broyden matrix (approximation of the Jacobian of $X_{c}$ with respect to $X_{f}$ ), and $C$ is an unknown constant. If we now assume that $B$ is the identity matrix, we can write directly:

$$
C=X_{c}^{d t}-X_{f}^{d t}
$$

Having an expression for $C$, we can now use again (4), and write:

$$
X_{f}^{t}=X_{c}^{t}-\left[X_{c}^{d t}-X_{f}^{d t}\right]=X_{c}^{t}-\left[X_{c}^{d t}-X_{c}^{t}\right]
$$

We now note that $X_{f}^{t}$ is the set of numerical values that will give the desired target response in the fine domain, and the expression between square brackets is the distance we discussed in the previous paragraph. In conclusion, starting with the target design in the coarse space $X_{c}^{t}$, we can obtain the target filter dimensions in the fine domain $X_{f}^{t}$ with only one (optimization) step, as long as the mapping between both model parameter spaces is linear and with a unitary Jacobian of $X_{c}$ with respect to $X_{f}$.

In the reminder of this paper we will show a number of examples that confirm these conclusions.

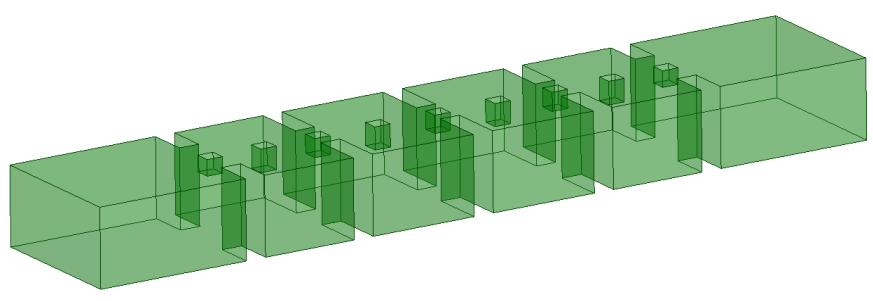

Fig. 10. Fully tunable four-pole filter structure.

\section{CAD DEMONSTRATION}

In this section, we will demonstrate that the One Step Aggressive Space Mapping (OS-ASM) behaviour anticipated in the previous section is indeed correct. This will be shown using a number of different microwave filters. Each filter will be simulated with FEST3D, as the LF space, and with HFSS from ANSYS as the HF space. The computational parameters used in FEST3D are as follows:

- Accessible modes $=5$

- Number of basis functions $=15$

- Green's function terms $=150$

- Stoping error criteria in optimizations $=0.001$

On the other hand, the HFSS computation was carried out with the following set of computational parameters:

- Minimum number of passes $=5$

- Maximum delta $S=0.001$

- Minimum convergent passes $=3$

thereby ensuring a high level of accuracy of the simulation results obtained. All the HF simulations will have the same simulation termination criteria, given by the maximum delta $S$ variance defined in the previous list for the HFSS computations.

In this context, it is important to note that the LF simulations carried out with FEST3D take, on average, less than 5 seconds, while the HF simulations carried out with HFSS take, on average, more than 15 minutes. For all computations we have used a PC with an Intel Core i7-6700 @ $3.4 \mathrm{GHz}$, and with a 16 GB RAM.

\section{A. Tunable Rectangular Four-pole Filter}

The first example that we will discuss is a fully tunable fourpole filter in rectangular waveguide. The target ideal structure is shown in Fig. 10. As we can see from Fig. 10, the structure contains rectangular tuning elements both in the apertures and in the resonant cavities. The filter specifications are as follows:

- Center frequency $=12 \mathrm{GHz}$

- Bandwidth = $200 \mathrm{MHz}$

- Return Loss $=25 \mathrm{~dB}$

The first step in the design procedure is to design a standard inductive rectangular waveguide filter with the same specifications. This is a convenient starting point, since filters of that type can be synthesized automatically using available commercial software packages. The next step is the transition from the standard inductive filter structure (not shown here for the sake of space) to the low fidelity (LF) model of the tunable configuration. The result obtained is shown in Fig. 11. 


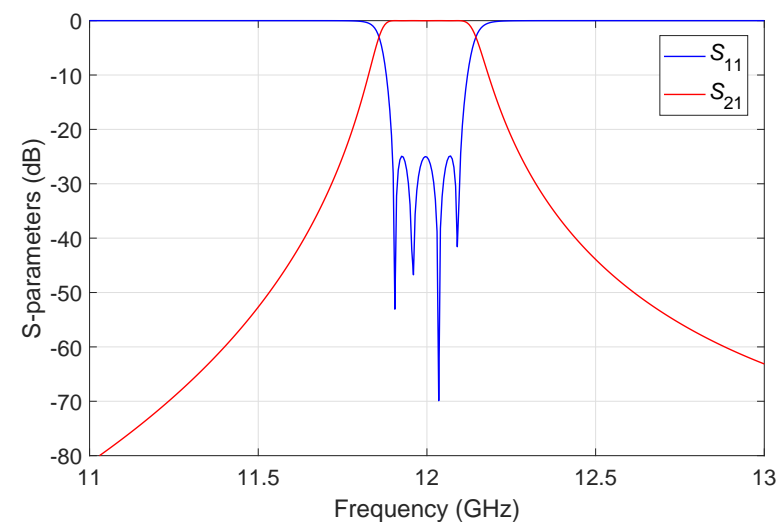

Fig. 11. Low-Fidelity fully tunable four-pole filter performance.

TABLE IV

DiMENSIONS OF THE FOUR-POLE STRUCTURE IN MILLIMETERS. ALL THE HEIGHTS ARE 9.525 MM.

\begin{tabular}{|c|c|c|}
\hline Structure & Width & Length \\
\hline I/O aperture & 8.826 & 2.5 \\
\hline First cavity & 19.05 & 12.811 \\
\hline Second aperture & 5.585 & 2.5 \\
\hline Second cavity & 19.05 & 14.297 \\
\hline Central aperture & 5.159 & 2.5 \\
\hline
\end{tabular}

The procedure that we have followed to perform the transition from the standard inductive rectangular waveguide filter to the structure in Fig. 10 is the one described in [22]. The final dimensions of the four-pole filter designed are shown in Table IV. Note that the filter is symmetric and all the screw penetrations are $2 \mathrm{~mm}$.

Now that we have obtained the target response in the LF space, we move to the HF space and perform another simulation using the same screw penetrations obtained with the LF model. Fig. 12 shows the comparison of the LF and HF results. As we expect, the performance of the two structures is different. We will now use the the ASM procedure to recover the HF performance in the LF space. Fig. 13 shows the results obtained.

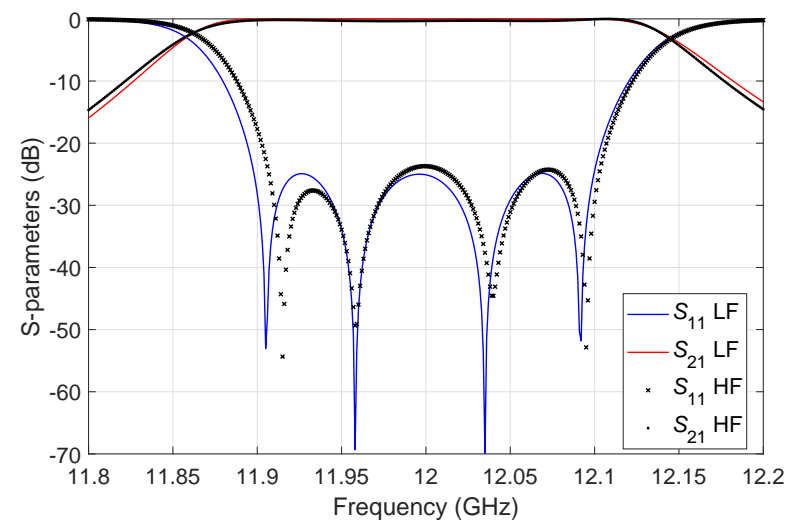

Fig. 12. Comparison of LF (blue) and HF (black dots) fully tunable four-pole filter responses.

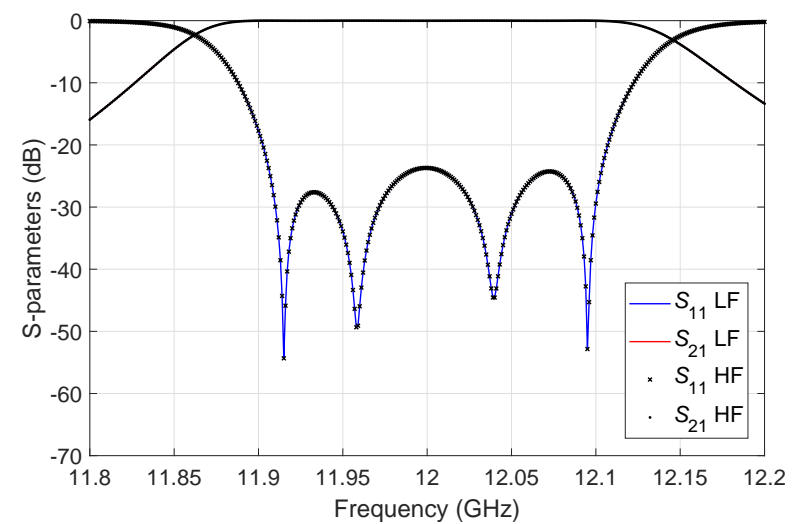

Fig. 13. HF and LF space alignment.

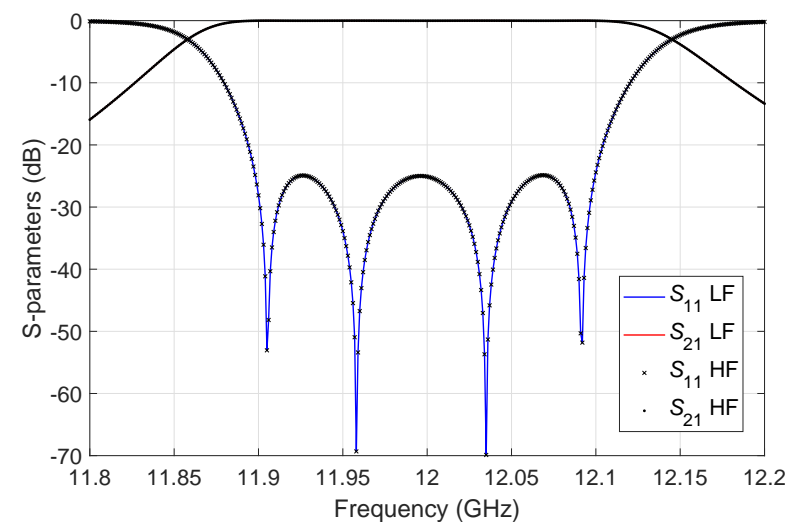

Fig. 14. HF final performance vs. LF performance.

As we can see the two curves are essentially identical. With this step we now have achieved a virtually perfect alignment between the HF and the LF spaces.

We will now measure the difference in screw penetration between the LF result in Fig. 13 and the HF result in Fig. 12 , and apply it in the opposite direction to the (full) HF model in Fig. 10. Fig. 14 shows the results obtained using the new set of screw penetrations compared with the target ideal response. As we can clearly see comparing Fig. 11 with Fig. 14 , the desired ideal performance has been essentially obtained in just one step.

This first example indeed demonstrates that convergence can be achieved in just one step. However, one can object that initial point (Fig. 13) is very close to the actual target. In the next example, to obtain a better demonstration of the OS-ASM behavior, we have significantly detuned the initial filter response in HFSS by changing the screw penetrations with random values. The initial detuned response that we have obtained with this change is shown in Fig. 15. Once we have this response we perform the ASM procedure again, recovering first the response with FEST3D and then applying the screw penetration changes to the HF space in the reverse direction. Fig. 16 shows the final result. As we can see, the filter performance has been fully recovered with only one step. 


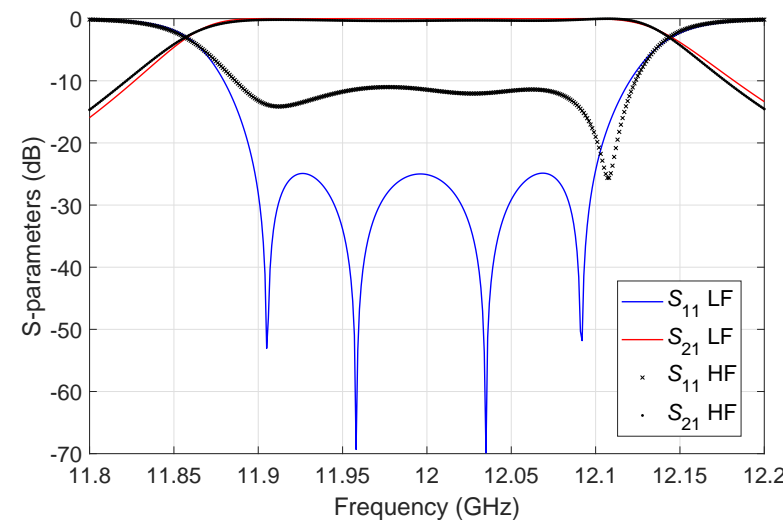

Fig. 15. Detuned HF performance with random values of tuning element penetration.

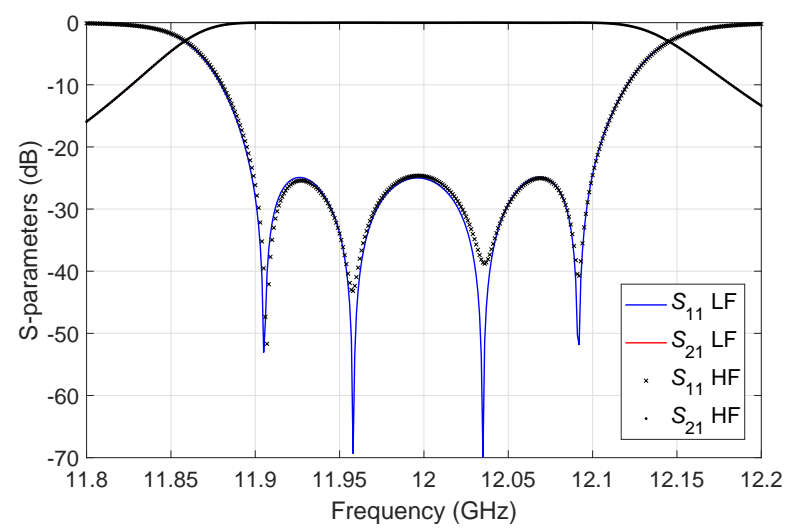

Fig. 16. HF model performance vs. LF performance.

\section{B. Tunable Rectangular Eight-pole Filter}

The filter used in the previous example was a simple fourpole filter. We will now demonstrate the OS-ASM with a more complex filter structure, namely, an eight-pole filter with the same center frequency and bandwidth of the four-pole filter. Following again the filter design procedure described in [22], we obtain the filter structure shown in Fig. 17, with the response shown in Fig. 18. The final dimensions of the eight-pole filter designed are shown in Table V. Note that the filter is symmetric and all the screws penetrate $2 \mathrm{~mm}$.

Next, we simulate the same structure in HFSS and we obtain the results shown in Fig. 19.

Once again, the filter performance is detuned. Following the same steps as the for first example, we apply the ASM procedure to recover the HF performance in the LF space.

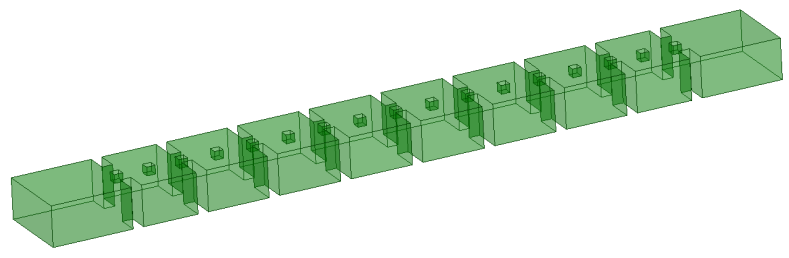

Fig. 17. Fully tunable eight-pole filter structure.

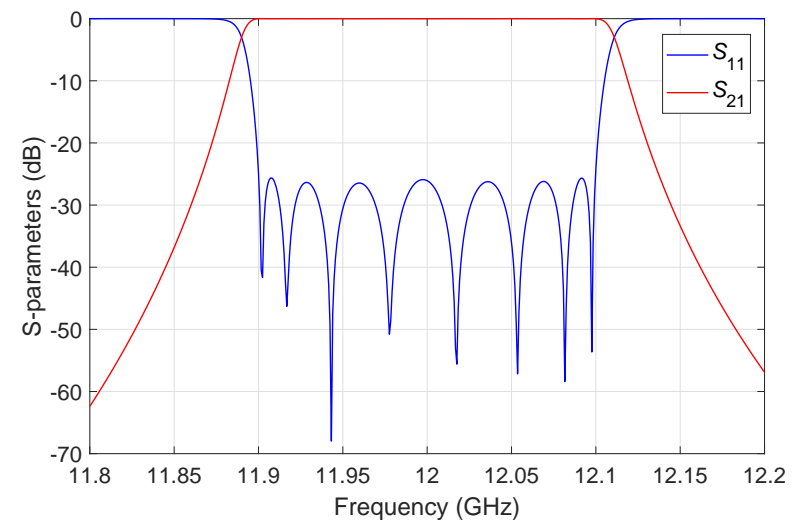

Fig. 18. LF model performance.

TABLE V

DiMENSIONS OF THE EIGHT-POLE STRUCTURE IN MILLIMETERS. ALL THE HEIGHTS ARE $9.525 \mathrm{MM}$.

\begin{tabular}{|c|c|c|}
\hline Structure & Width & Length \\
\hline I/O aperture & 8.694 & 2.5 \\
\hline First cavity & 19.05 & 12.938 \\
\hline Second aperture & 5.379 & 2.5 \\
\hline Second cavity & 19.05 & 14.407 \\
\hline Third aperture & 4.844 & 2.5 \\
\hline Third cavity & 19.05 & 14.551 \\
\hline Fourth aperture & 4.738 & 2.5 \\
\hline Fourth cavity & 19.05 & 14.577 \\
\hline Central aperture & 4.715 & 2.5 \\
\hline
\end{tabular}

Fig. 20, shows the result after only one step. As we can see, the target performance has been obtained again in just one step.

Similarly to what we have done in the previous example, we next detuned the filter performance changing the screw penetrations by random values. Fig. 21 shows the detuned response of the filter, and Fig. 22 the result of the application of the one step space mapping procedure. As we can clearly see, we have obtained the ideal target response of the filter in the HF space in just one step.

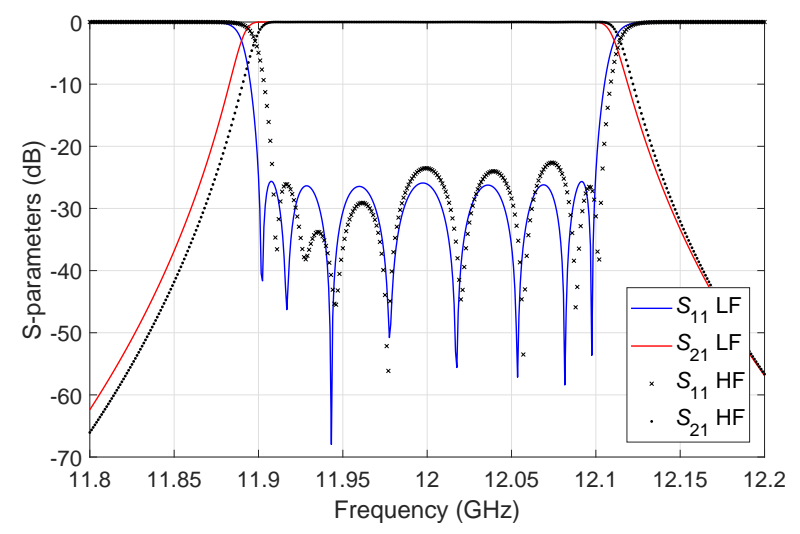

Fig. 19. HF model initial performance vs. LF performance. 


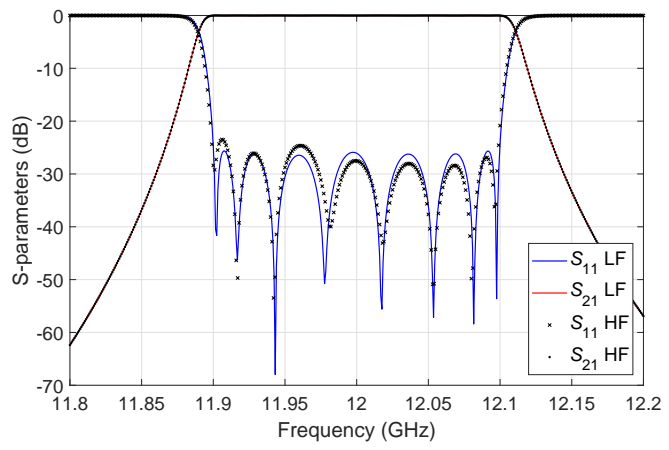

Fig. 20. Result of the first iteration ASM procedure vs. LF performance.

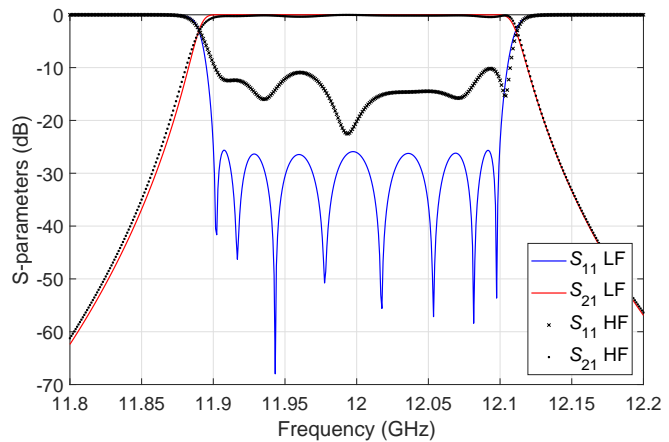

Fig. 21. HF model detuned response vs. LF performance.

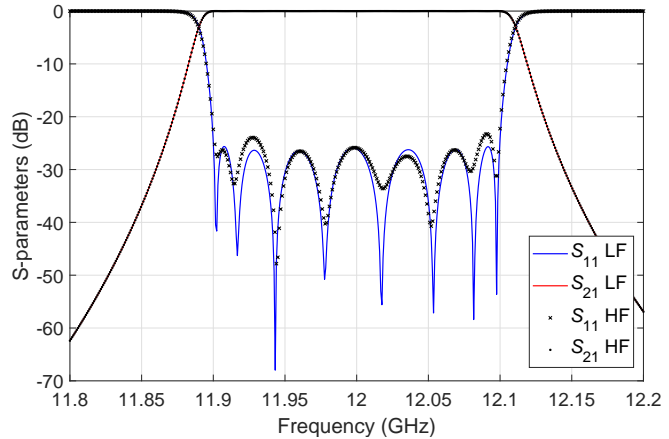

Fig. 22. Result of the first iteration ASM procedure vs. LF performance.

\section{Classic Circular Dual-mode Filter}

The next structure that we discuss is a substantially more complex filter, namely, a classic circular waveguide dualmode filter. The filter structure is shown in Fig. 23. The filter specifications are as follows:

- Center frequency $=17.59 \mathrm{GHz}$

- Bandwidth $=36 \mathrm{MHz}$

- Return Loss $=20 \mathrm{~dB}$

The final dimensions of the classic dual-mode filter designed are shown in Table VI. Note that the filter is symmetric and the I/O rectangular waveguide is the standard WR-75.

The screw penetrations are $1.848 \mathrm{~mm}, 1.278 \mathrm{~mm}$ and 1.474 $\mathrm{mm}$ for the $0^{\circ}, 45^{\circ}$, and $90^{\circ}$ oriented screws in the circular waveguide, respectively.
TABLE VI

DIMENSIONS OF THE CLASSIC DUAL-MODE FILTER STRUCTURE IN MILLIMETERS.

\begin{tabular}{|c|c|c|c|c|}
\hline Structure & Height & Width & Length & Radius \\
\hline I/O aperture & 2 & 6.262 & 1 & - \\
\hline Circular cavity & - & - & 32.133 & 7.959 \\
\hline Central vertical aperture & 4.443 & 1 & 1 & - \\
\hline Central horizontal aperture & 1 & 2.833 & 1 & - \\
\hline
\end{tabular}

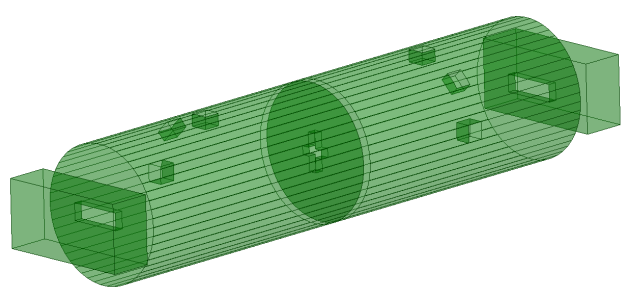

Fig. 23. Classic circular waveguide dual-mode filter structure.

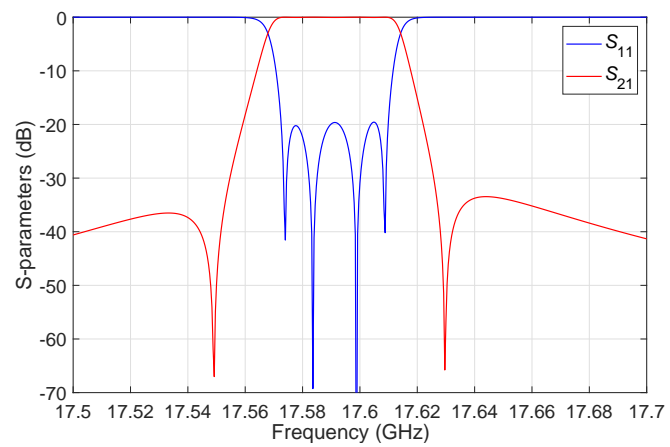

Fig. 24. Response of the dual-mode filter.

The filter response in Fig. 24 has been obtained with FEST3D using the same set of computational parameters as for the previous examples in the LF space. Simulating with HFSS (HF) the structure we obtain the result shown in Fig. 25 with black dots. Now we can apply the first step of the SM procedure and recover the HF performance in the LF space. Fig. 25 shows the differences between the LF and HF spaces, and Fig. 26 the final HF performance obtained by changing only the screws and aperture dimensions in the opposite direction.

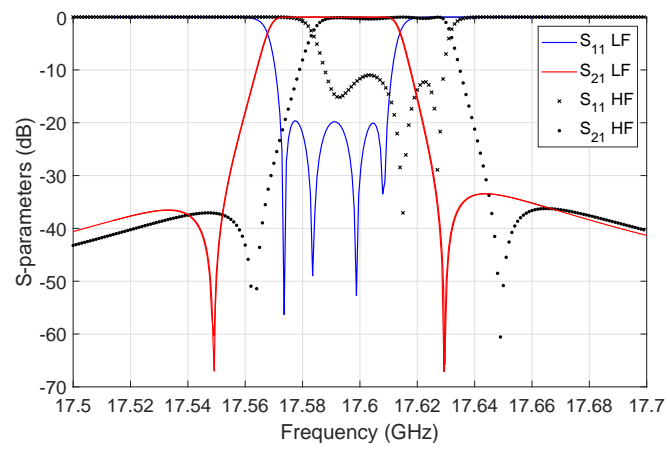

Fig. 25. Initial HF response vs. LF performance. 


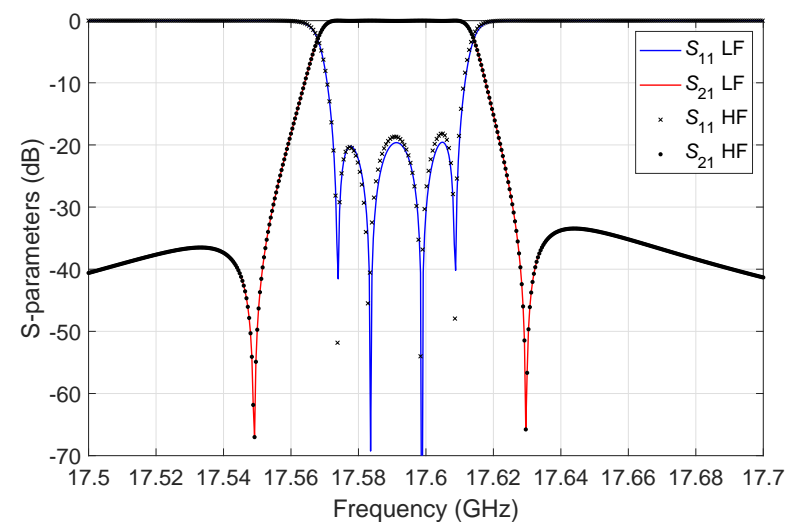

Fig. 26. Final HF filter performance vs. LF performance.

As we can see, we have again obtained the desired response in the HF space with only one SM step.

\section{More General Examples}

Up to now, we have demonstrated the OS-ASM procedure with different filters where in both LF and HF spaces the basic filter structures are almost exactly identical. In real filter design, however, the final structures are always affected by manufacturing errors.

Furthermore, the basic filter structure is usually slightly modified to reduce manufacturing costs (rounded corners), and real cylindrical screws are used as tuning elements instead of square cylinders. In practice therefore, even though the filter design may always start with an ideal structure, the final hardware will inevitably have some small geometrical differences with respect to the ideal starting point. One fact, however, will be unchanged. If the ideal structure and the final structure must have the same identical electrical performance, they must also have the same amount of stored electrical and magnetic energy. Furthermore, the perturbation theory must always be applicable to both structures. It is therefore interesting to see what is the effect on the ASM procedure if the LF and HF structures are not exactly identical.

\section{A. Tunable Rectangular Four-pole Filter with Rounded Cor- ners}

As a first example we will use as the HF filter the (real) structure in Fig. 27 with rounded corners of $2 \mathrm{~mm}$. Fig. 28 shows the initial response, obtained with HFSS, if we use as the starting point the structure with sharp corners and square tuning elements that we have used as a previous example (see Fig. 10) in section VI.

As we can clearly see, the filter response is now very strongly detuned. The performance of the filter obtained after the first ASM iteration is shown in Fig. 29.

As we can see, in this case we have not recovered the ideal filter performance yet. We need to apply the procedure again (without updating the mapping matrix) in order to recover the ideal response. Fig. 30 shows the result of this second iteration.

As we can see, with just two iterations we have fully recovered the filter performance.

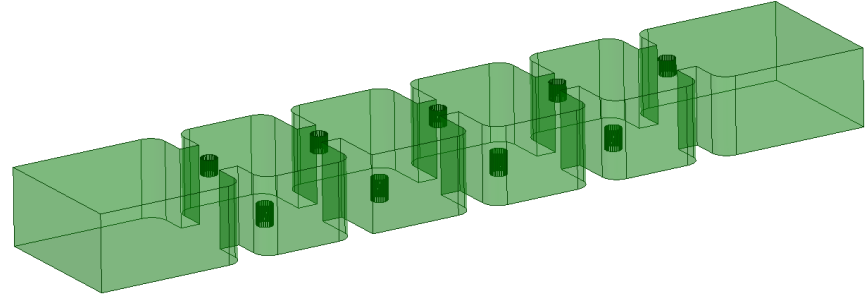

Fig. 27. HF model structure with rounded corners and circular screws.

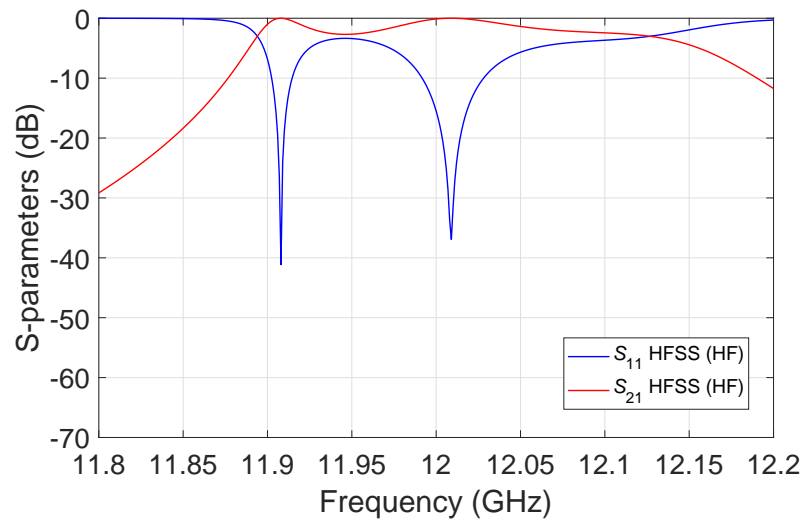

Fig. 28. Initial response of the HF model.

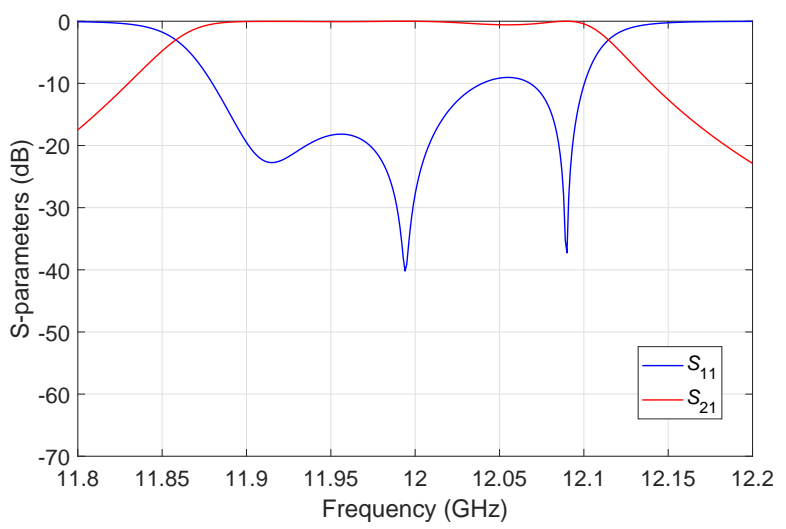

Fig. 29. Result of the first iteration in HF.

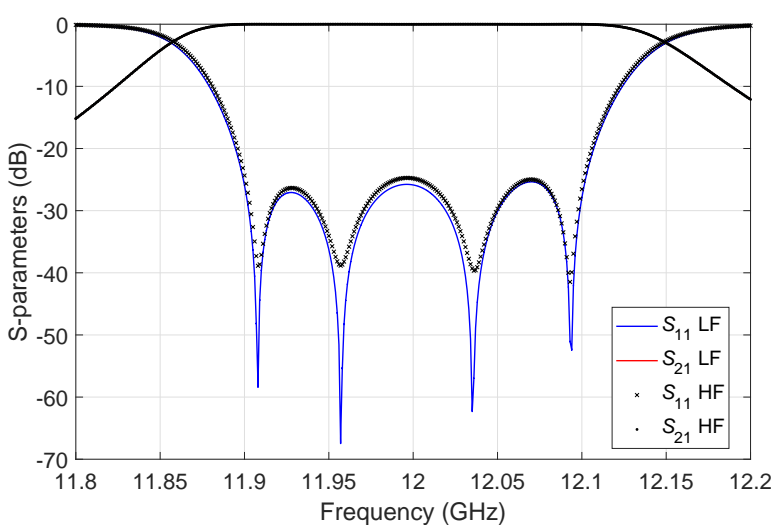

Fig. 30. Result of the second iteration in HF vs. ideal response. 


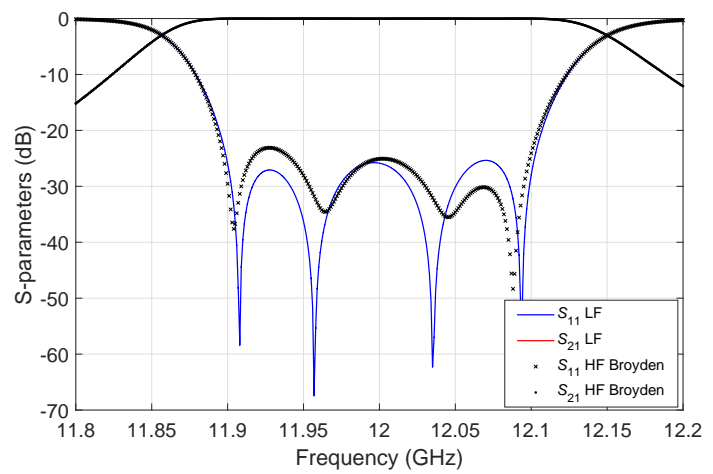

Fig. 31. Result of the second iteration with Broyden vs. ideal response.

It is important to note in this context that in this example we have stopped the ASM procedure (the exit condition) when the error given by the optimizer in FEST between the target performance and the last HF response is less or equal to one. The same exit condition has been used in all other examples requiring several ASM iterations.

At this point we must raise an important question: what happens if we apply the classic ASM procedure based on the use of the Broyden update, instead of using the identity matrix in the whole process (as we have done so far)?

In the next section we have indeed performed this test in order to compare the results obtained.

\section{B. Tunable Rectangular Four-pole Filter with ASM and Broy- den Update}

The initial and the first step of the Broyden based ASM procedure will produce the same results shown in Fig. 28 and Fig. 29. After the first two HF computations, we can use the Broyden formula and obtain the result shown in Fig. 31.

As we can see, using the Broyden based ASM procedure we have essentially recovered the ideal filter response. However, if we compare this result with the one shown in Fig. 30, we can clearly see that the performance using Broyden is less accurate. From the results obtained in this experiment, therefore, it appears that, even though the LF and HF structures are not exactly identical, it is more efficient to apply the ASM with the identity matrix as the space mapping matrix, instead of updating it by the Broyden formula in every step.

\section{Fully Tunable Dual-mode Filter}

The second filter to be designed with rounded corners and tuning screws will be the fully tunable dual-mode filter discussed in [23]. In this case, all rounded corners have $1 \mathrm{~mm}$ curvature radius. Fig. 32 and Fig. 33 shows the ideal filter structure (with sharp corners), and the desired response.

The software tool HFSS is again used as the HF space, together with the structure shown in Fig. 32. Initially, we use in the HF space the same screw penetrations as the ideal model but using now rounded corners. Its response is shown in Fig. 34 with black dots.

We next apply the ASM procedure. Figs. 34, 35 and 36 shows the initial response compared with the ideal one, and

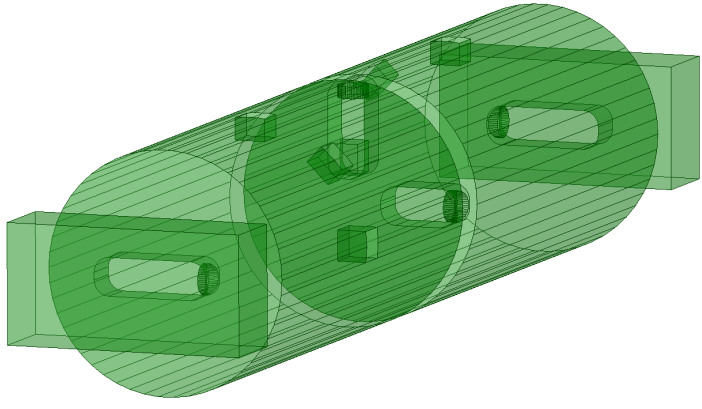

Fig. 32. Fully tunable dual-mode filter structure in LF.

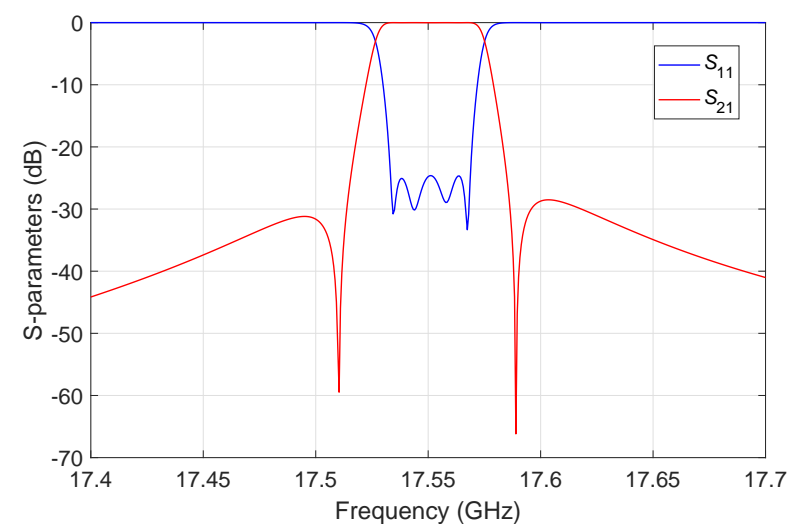

Fig. 33. Ideal LF filter response.

the next iterations of the process using the identity mapping matrix. Fig. 37 shows the final performance of the HF filter.

In this case, we have performed 3 iterations of the ASM procedure to obtain the desired response, always using the identity matrix instead of the Broyden update formula for the space mapping matrix.

\section{Fully Tunable Dual-mode Filter with Broyden Based ASM}

We now perform again the design procedure updating this time the space mapping matrix with the Broyden formula in every additional ASM iteration. The first iteration gives the result already discussed.

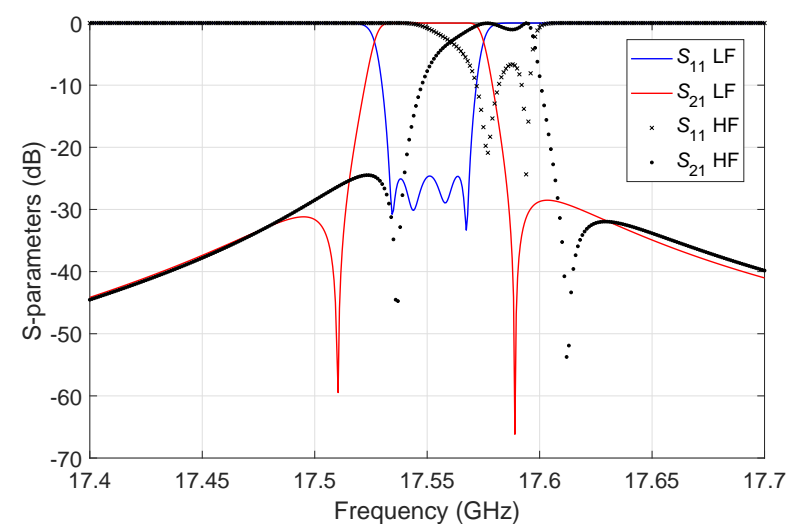

Fig. 34. Comparison between initial HF performance and LF performance. 


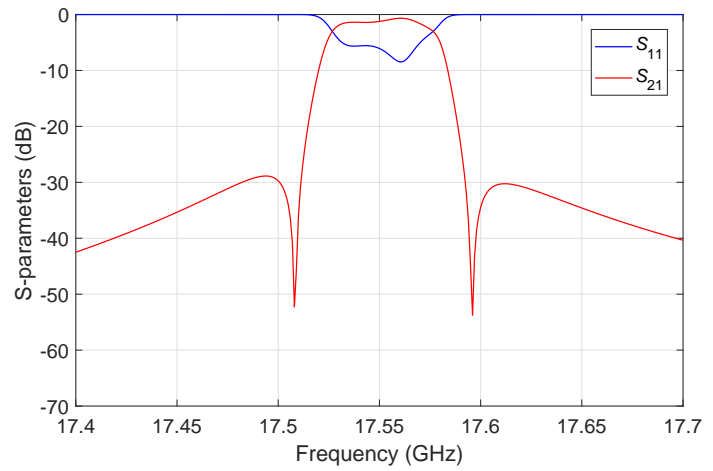

Fig. 35. Result of first iteration in HF.

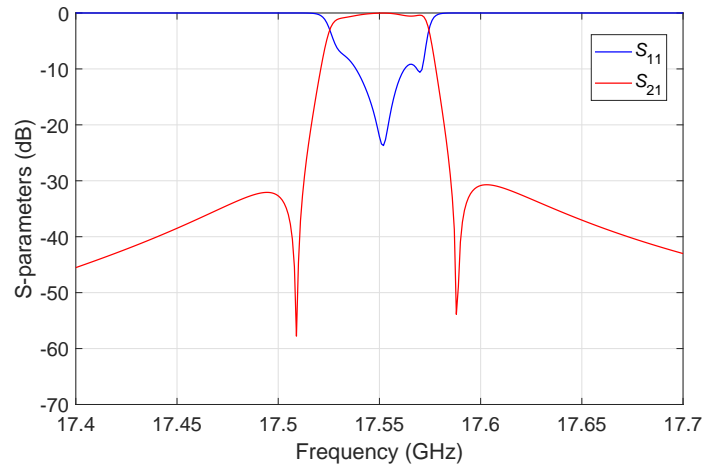

Fig. 36. Result of second iteration in HF.

We therefore start from the result of the first iteration, and recover the response shown in Fig. 35 and we apply the Broyden formula. The performance obtained is shown in Fig. 38.

We must now perform another iteration, obtaining the response shown in Fig. 39. Finally, after four iterations we obtain the desired response shown in Fig. 40.

Comparing this result with the previous result using the identity mapping matrix for all the iterations, updating the space mapping matrix with the Broyden formula requires one more optimization step to recover the desired response.

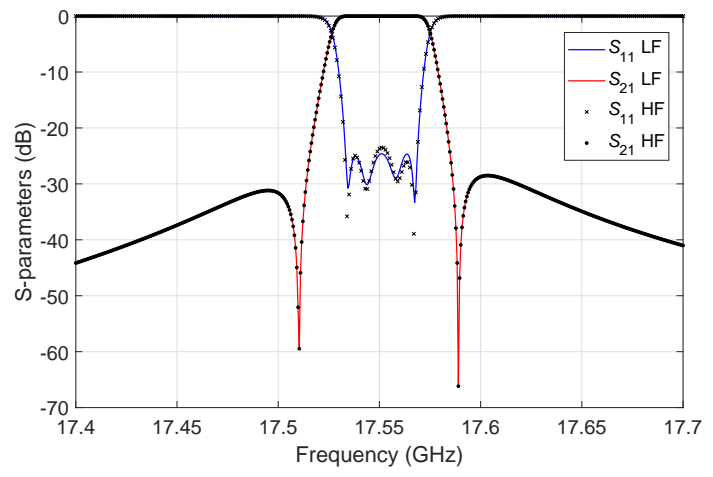

Fig. 37. Final HF filter performance of the third iteration vs. ideal response.

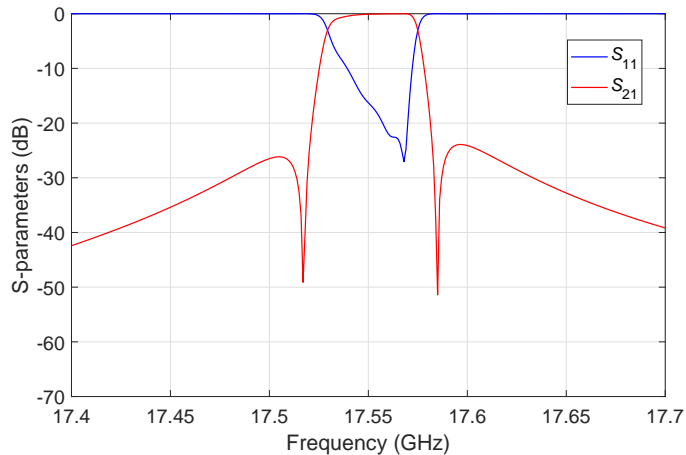

Fig. 38. Response of the second iteration with Broyden formula in HF.

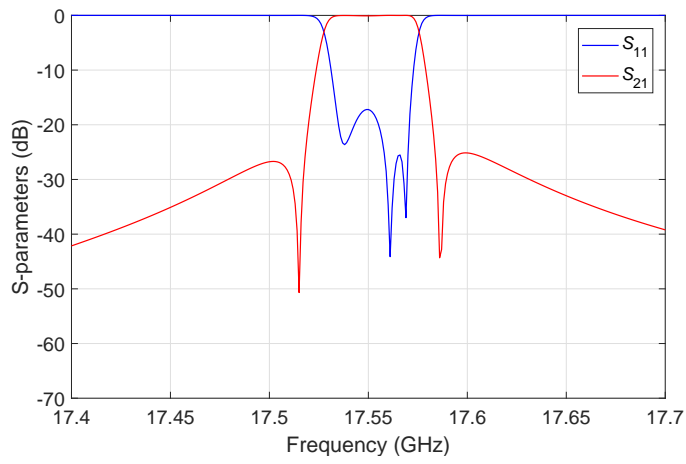

Fig. 39. Response of the third iteration with Broyden formula in HF.

\section{E. Folded Rectangular Waveguide Tunable Filter}

The last example that we discuss is the design of a tunable folded filter in rectangular waveguide [24]. The design parameters are:

- Center frequency $=17.3 \mathrm{GHz}$

- Bandwidth $=36 \mathrm{MHz}$

- Return Loss $=25 \mathrm{~dB}$

Following the cavity numeration of [24], Table VII shows the dimensions of the filter structure and the initial screw penetrations. Note that, in this case, the I/O waveguide is the standard WR-51 and rounded corners of radius $2 \mathrm{~mm}$ have been considered.

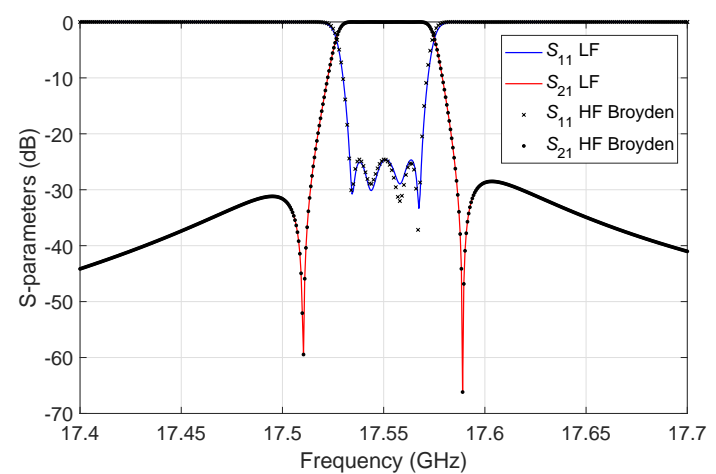

Fig. 40. Result of the fourth iteration with Broyden in HF filter vs. ideal response. 
TABLE VII

DiMENSIONS OF THE FOLDED FILTER STRUCTURE (MM). ALL THE HEIGHTS ARE $6.477 \mathrm{MM}$.

\begin{tabular}{|c|c|c|c|}
\hline Structure & Width & Length & Screw penetration \\
\hline Input aperture & 6.5 & 3.469 & 0.633 \\
\hline First cavity & 12.954 & 30.543 & 1.965 \\
\hline Second aperture & 3.899 & 2.654 & 1.413 \\
\hline Second cavity & 12.954 & 33.106 & 1.913 \\
\hline Third aperture & 3.990 & 3.117 & 1.822 \\
\hline Third cavity & 12.954 & 33.134 & 1.898 \\
\hline Fourth aperture & 3.845 & 2.654 & 1.077 \\
\hline Fourth cavity & 12.954 & 30.982 & 2.002 \\
\hline Fifth aperture & 6.725 & 16.315 & 2.024 \\
\hline Output aperture & 6.5 & 3.671 & 1.401 \\
\hline
\end{tabular}

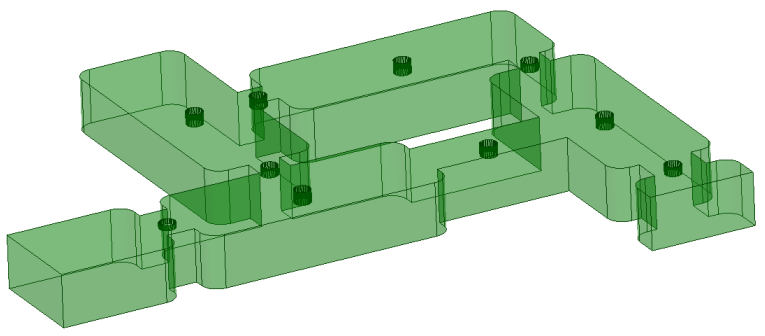

Fig. 41. Filter structure.

The filter structure in the LF space is shown in Fig. 41 (with sharp corners) and its performance in Fig. 42.

The initial HF performance using the same tuning screw penetrations as in the LF space is shown in Fig. 43 with black dots.

We next apply the ASM procedure to recover the desired (ideal) response in the HF space. Figs. 43, 44 and 45 shows the initial comparison and the next iterations of the process. Fig. 46 shows the final performance of the HF filter obtained with only three iterations.

\section{F. Folded Rectangular Waveguide Tunable Filter with Broyden Based ASM}

We now perform again the same basic ASM design procedure, but, this time, we update the space mapping matrix with the Broyden formula.

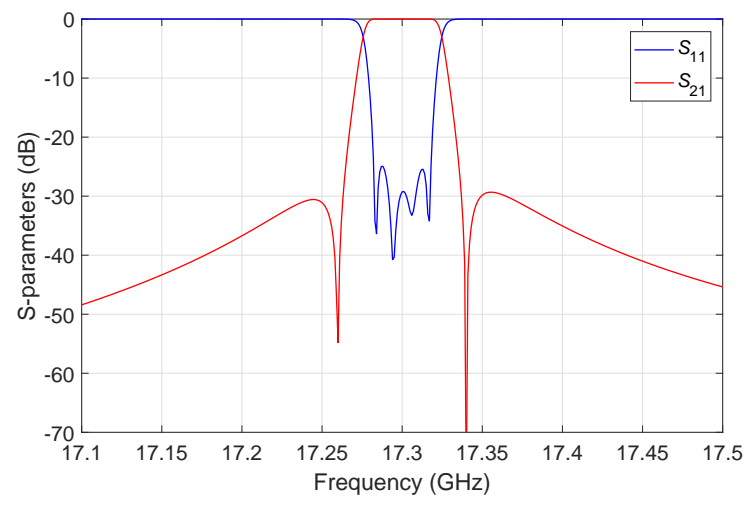

Fig. 42. Ideal LF filter response.

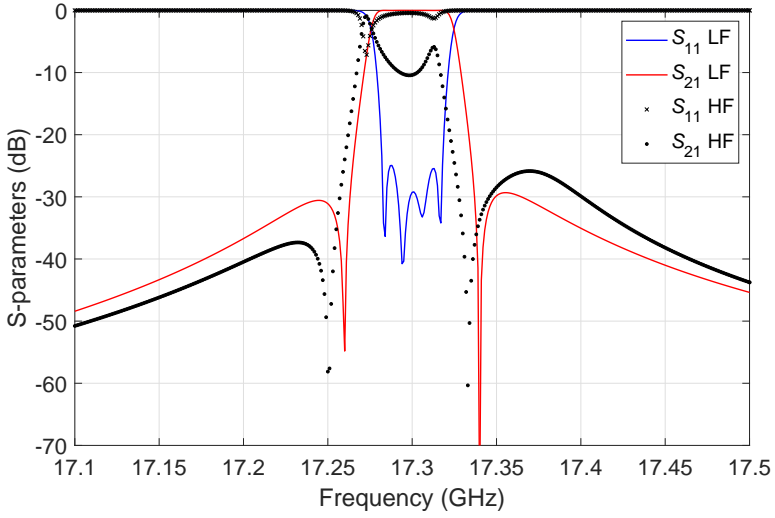

Fig. 43. Comparison between initial HF performance and LF performance.

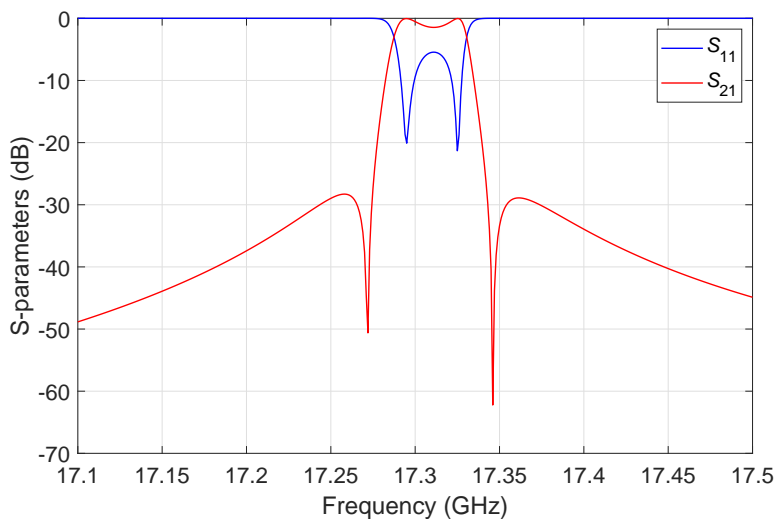

Fig. 44. Result of the first iteration in HF.

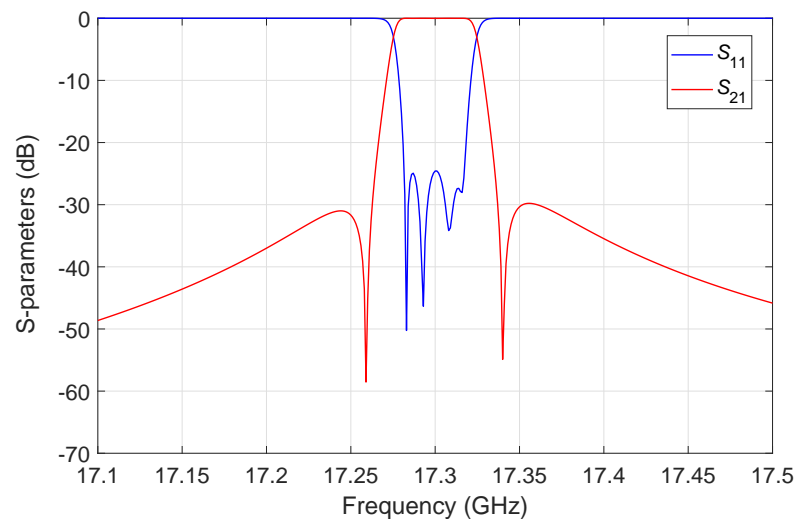

Fig. 45. Result of the second iteration in HF. 


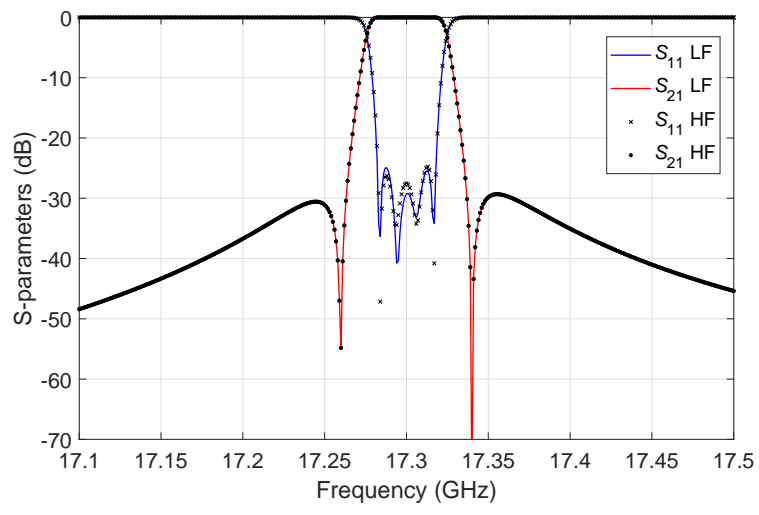

Fig. 46. Result of the third iteration in HF filter vs. ideal response.

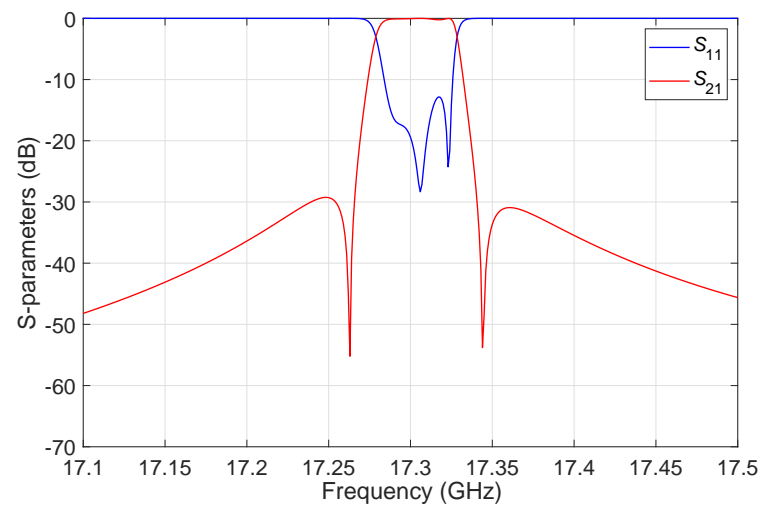

Fig. 47. Response of the second iteration with Broyden formula in HF.

We can start with the second iteration of the process. Figs. 47 and 48 shows the different results obtained, and Fig. 49 shown the final performance. The final result has been obtained with four iterations.

\section{Definition of Space Alignment}

Based on all of the above discussion and results, we are now going to propose a definition of alignment between the LF and the HF spaces in the context of ASM.

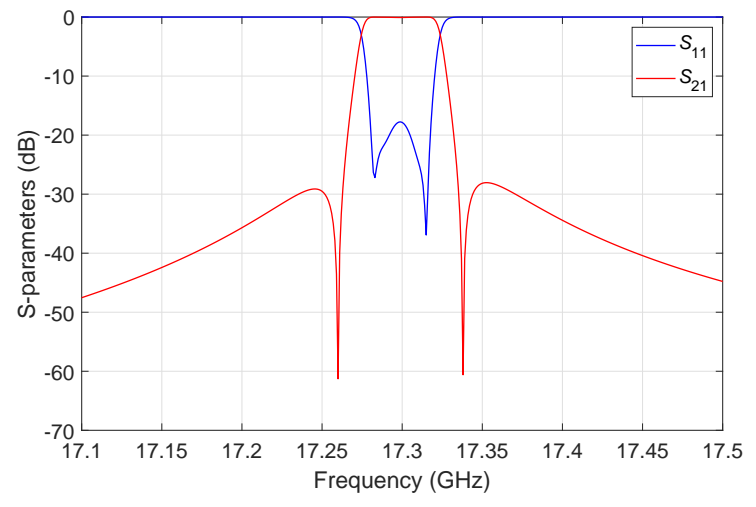

Fig. 48. Response of the third iteration with Broyden formula in HF.

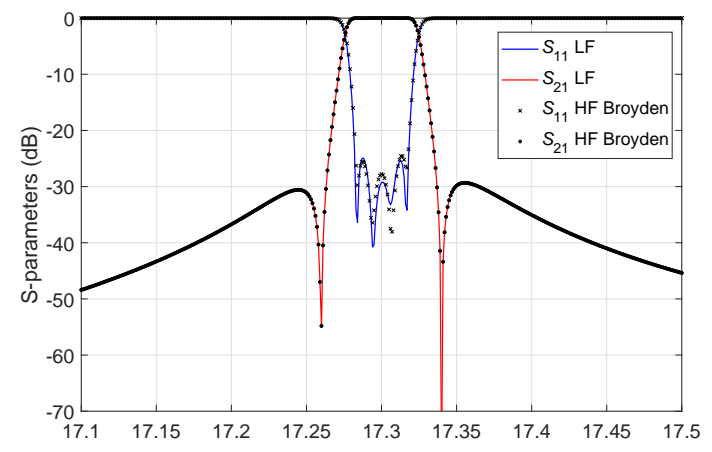

Fig. 49. Response of the fourth iteration with the Broyden formula in HF vs. ideal response.

Two spaces are considered to be perfectly aligned if and only if the following two conditions are satisfied:

1) Two points in the respective parameter spaces can be found that produce the same identical responses (points of alignment).

2) The derivatives (or the Jacobians) of the responses with respect to corresponding physical parameters in the two spaces, evaluated at the points of alignment, are identical.

Our investigations clearly indicate that, if the two conditions above are satisfied, and the LF and HF structures are identical, we can expect an ASM procedure to converge to the desired performance in just one step.

\section{CONCLUSION}

In the context of microwave waveguide filter design and optimization based on ASM, the choice of the LF and HF spaces (structures, or models) is indeed a key point. Naturally, the HF model must be as close as possible to physical reality. The LF model, on the other hand, must be able to represent the same physical reality, in particular with respect to the tuning elements, but needs to produce results with a significantly higher computational efficiency. As we have clearly shown in this paper, the fact that both models must obey the perturbation theory will ensure that the ASM procedure will converge in just one step if the LF and HF structures are physically identical. In the case in which the LF and HF physical structure are not exactly identical, but differ from each other in relatively small volumes, our investigation clearly shows that the ASM based on the identity space mapping matrix is more efficient than the classic ASM based on the Broyden update formula. Finally, in the case in which the LF and HF models are established in spaces that are not (physically) congruent (i.e., the modeling of a filter using inverters and transmission lines as the LF space, and with full-wave EM software tools as the HF space), we expect that the ASM procedure will converge more efficiently with the help of the Broyden update, so that a proper mapping between the two (very different) spaces can be established faster. 


\section{REFERENCES}

[1] J. W. Bandler, R. M. Biernacki, S. H. Chen, P. A. Grobelny, and R. H. Hemmers, "Space mapping technique for electromagnetic optimization," IEEE Trans. Microw. Theory Tech., vol. 42, no. 12, pp. 2536 - 2544, Dec. 1994.

[2] J. W. Bandler, R. M. Biernacki, S. H. Chen, R. H. Hemmers, and K. Madsen, "Electromagnetic optimization exploiting aggressive space mapping," IEEE Trans. Microw. Theory Tech., vol. 43, no. 12, pp. 2874 - 2882, Dec. 1995

[3] C. G. Broyden, "A class of methods for solving nonlinear simultaneous equations," Math. Comp., vol. 19, pp. 577 - 593, 1965.

[4] J. W. Bandler, Q. S. Cheng, S. A. Dakroury, A. S. Mohamed, M. H. Bakr, K. Madsen, and J. Søndergaard, "Space mapping: the state of the art," IEEE Trans. Microw. Theory Tech., vol. 52, no. 1, pp 337 - 361, Jan. 2004.

[5] J. E. Rayas-Sanchez, "Power in simplicity with ASM: tracing the aggressive space mapping algorithm over two decades of development and engineering applications," IEEE Microw. Mag., vol. 17, no. 4, pp. 64 76, Mar. 2016.

[6] J.W. Bandler, R.M. Biernacki, and S.H. Chen, "Space mapping optimization of waveguide filters using finite element and mode-matching electromagnetic simulators," IEEE MTT-S Int. Microw. Symp. Dig., 1997, vol. 2, pp. $635-638$.

[7] J.W. Bandler, R.M. Biernacki, and S.H. Chen, "Space mapping optimization of waveguide filters using finite element and mode-matching electromagnetic simulators," Int. Journal of RF and Microwave Computer-Aided Engineering, vol. 9, no. 1, pp. 54 - 70, Jan. 1999.

[8] M. A. Ismail, D. Smith, A. Panariello, Y. Wang, and M. Yu, "EM-based design of large-scale dielectric-resonator filters and multiplexers by space mapping," IEEE Trans. Microw. Theory Tech., vol. 52, no. 1, pp. 86 - 92, Jan. 2004.

[9] R. Lehmensiek and M. Petrie, "An efficient adaptive frequency sampling algorithm for model-based parameter estimation as applied to aggressive space mapping," Microw. and Opt. Tech. Lett., vol. 24, no. 1, pp. 71 78, Nov. 1999.

[10] M. A. Ismail, K. G. Engel, and Y. Ming, "Multiple space mapping for RF T-switch design," IEEE MTT-S Int. Microw. Symp. Dig., Fort Worth, TX, June 2004, pp. 1569 - 1572.

[11] J. Morro et all., "Fast automated design of waveguide filters using aggressive space mapping with a new segmentation strategy and a hybrid optimization algorithm," IEEE Trans. Microw. Theory Tech., vol. 53, no. 4, pp. 1130 - 1142. Apr. 2005.

[12] E. Diaz, J. Morro, A. Belenguer, H. Esteban, and V. E. Boria, "CAD technique for designing H-plane waveguide filters considering rounded corners," IEEE MTT-S Int. Microw. Symp. Dig., 2013, pp. 1 - 3.

[13] M. Brumos, V. E. Boria, and M. Guglielmi, "Correction of manufacturing deviations in circular-waveguide dual-mode filters using aggressive space mapping," Proc. of the 44th European Microw. Conf., 2014, pp. $624-627$.

[14] G. Crevecoeur, L. Dupr, L. Vandenbossche, and R. Van de Walleb, "Reconstruction of local magnetic properties of steel sheets by needle probe methods using space mapping techniques," J. Appl. Phys., vol. 99 , pp. 08H905-08H905-3, Apr. 2006.

[15] J. Wu, Y. Dong, J. He, and J. Deng, "An ameliorated aggressive space mapping based on responses error," Proc. Int. Workshop Microwave Millimeter Wave Circuits System Technology, Chengdu, China, Apr. 2012, pp. 14.

[16] J. Xu, X. Luo, L. Z. Cao, and R. S. Chen, "Optimization of coaxial dielectric resonator filter with aggressive space mapping," Proc. AsiaPacific Microw. Conf., Kaohsiung, Taiwan, Dec. 2012, pp. 229 - 231.

[17] Q. Deng, T. Fan, and S. Lu, "The design of Ka band filter with active space mapping algorithm," Proc. Asia-Pacific Conf. Antennas Propagation, Harbin, China, July 2014, pp. 11401143.

[18] A. Rodriguez et all., "Robust optimization and tuning of microwave filters and artificial transmission lines using aggressive space mapping techniques," IEEE MTT-S Int. Microw. Symp., 2017, pp. 1501 - 1504.

[19] J. Ossorio, J. Vague, V. E. Boria, and M. Guglielmi, "Efficient implementation of the aggressive space mapping technique for microwave filter design," Proc. of the 47th European Microw. Conf., 2017, pp. 644 - 647.

[20] G. Conciauro, M. Guglielmi, and R. Sorrentino, Advanced Modal Analysis, Wiley, 2000.

[21] A. Cunliffe and L. E. S. Mathias, "Some perturbation effects in cavity resonators," Proc. of the IEEE - Part III: Radio and Communication Engineering, vol. 1950, no. 10, pp. 367 - 376, Oct. 1950.

[22] M. Guglielmi and A. A. Melcon, "Novel design procedure for microwave filters," Proc. 23rd European Microw. Conf., 1993, pp. 212 - 213.
[23] J. Ossorio, J. Vague, V. E. Boria, and M. Guglielmi, "Exploring the tunability range of classic circular waveguide dual-mode filters using EM-based CAD," IEEE MTT-S Int. Conf. Numerical Electromagnetic and Multiphysics Modeling and Optimization for RF, Microwave, and Terahertz Applications (NEMO), May 2017, pp 332 - 334.

[24] J. Ossorio, J. Vague, V. E. Boria, and M. Guglielmi, "Exploring the tuning range of channel filters for satellite applications using electromagnetic-based computer aided design tools," IEEE Trans. Microw. Theory Tech., no. 9, pp. 1 - 9, Nov. 2017.

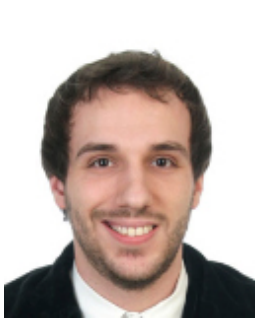

Javier Ossorio was born in Valencia, Spain on March 24, 1992. He received the degree and double master in telecommunications engineering from the Polytechnic University of Valencia, Spain, in 2014 and 2016, respectively. He is currently a Ph.D student in telecommunications in the iTEAM group at Polytechnic University of Valencia. His current research activities includes EM simulations, efficient design and optimizations of waveguide filters and development of new tunable structure filters.

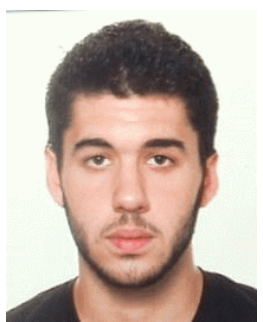

Juan Carlos Melgarejo was born in Alicante, Spain in 1993. He obtained his bachelors degree in Telecommunications from the Universitat Politcnica de Valncia (UPV) in 2015. From there, he continued his studies with the UPV where he pursued a double Masters in Telecommunications Systems. In 2017, he began his Ph.D. at the university where his main research interests have been investigating microwave passive devices and new manufacturing techniques for satellite components.

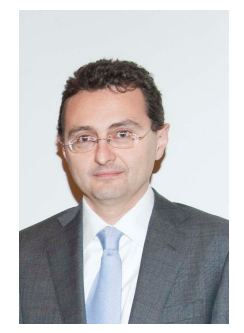

Vicente E. Boria (S'91-A'99-SM'02-F' 18) was born in Valencia, Spain, on May 18, 1970. He received his Ingeniero de Telecomunicacin degree (with firstclass honors) and the Doctor Ingeniero de Telecomunicacin degree from the Universidad Politcnica de Valencia, Valencia, Spain, in 1993 and 1997, respectively. In 1993 he joined the Departamento de Comunicaciones, Universidad Politcnica de Valencia, where he has been Full Professor since 2003. In 1995 and 1996, he was holding a Spanish Trainee position with the European Space Research and Technology Centre, European Space Agency (ESTEC-ESA), Noordwijk, The Netherlands, where he was involved in the area of EM analysis and design of passive waveguide devices. He has authored or co-authored 10 chapters in technical textbooks, 180 papers in refereed international technical journals, and over 200 papers in international conference proceedings. His current research interests are focused on the analysis and automated design of passive components, left-handed and periodic structures, as well as on the simulation and measurement of power effects in passive waveguide systems. Dr. Boria has been a member of the IEEE Microwave Theory and Techniques Society (IEEE MTT-S) and the IEEE Antennas and Propagation Society (IEEE AP-S) since 1992. He acts as a regular reviewer of the most relevant IEEE and IET technical journals on his areas of interest. Presently, he serves as Associate Editor of IEEE Microwave and Wireless Components Letters and IET Electronics Letters, and as Editorial Board member of International Journal of $\mathrm{RF}$ and Microwave Computer-Aided Engineering. He is also member of the Technical Committees of the IEEE-MTT International Microwave Symposium and of the European Microwave Conference. 


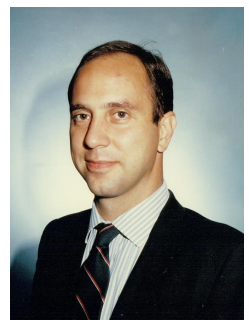

Marco Guglielmi was born in Rome, Italy, on December 17, 1954. He received the degree Laurea in Ingegneria Elettronica in 1979 from the University of Rome La Sapienza, Rome, Italy, where in 1980 he also attended the Scuola di Specializzazione in Elettromagnetismo Applicato. In 1981 he was awarded a Fulbright Scholarship in Rome, Italy, and an HISP (Halsey International Scholarship Programme) from the University of Bridgeport, Bridgeport, Connecticut, USA, where in 1982 he obtained an MS Degree in Electrical Engineering. In 1986 he received a PhD degree in Electrophysics from the Polytechnic University, Brooklyn, New York, USA. From 1984 to 1986 he was Academic Associate at Polytechnic University, and from 1986 to 1988 he was Assistant Professor in the same institution. From 1988 to 1989 he was Assistant Professor at the New Jersey Institute of Technology, Newark, New Jersey, USA. In 1989 he joined the European Space Agency as a Senior Microwave Engineer in the RF System Division of the European Space Research and Technology Centre (ESTEC), Noordwijk, The Netherlands, where he was in charge of the development of microwave filters and electromagnetic simulation tools. In 2001 he was appointed Head of the Technology Strategy Section of ESTEC where he contributed to the development of management processes and tools for the formulation of a European strategy for Space Technology Research and Development. In 2014 Dr. Guglielmi retired from the European Space Agency and is currently holding the position of Invited Senior Researcher at the Polytechnic University of Valencia, Valencia, Spain. Dr. Guglielmi has been elevated to the grade of Fellow of the IEEE in January 2013 For contributions to multimode equivalent networks and microwave filter design.

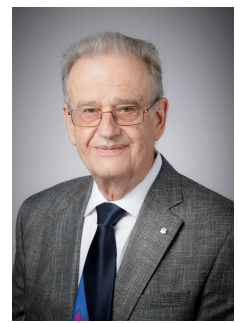

John W. Bandler (S'66-M'66-SM'74-F'78-LF'06) studied at Imperial College, London, England, and received the B.Sc.(Eng.), Ph.D., and D.Sc.(Eng.) degrees from the University of London, England, in 1963, 1967, and 1976, respectively. He joined McMaster University, Canada, in 1969. He is now a Professor Emeritus. He founded Optimization Systems Associates Inc. in 1983 and sold it to HewlettPackard in 1997. He is President of Bandler Corporation, Dundas, Ontario, Canada. Dr. Bandler is a Fellow of several societies, including the Royal Society of Canada and the Canadian Academy of Engineering. In 2004, he received the IEEE MTT-S Microwave Application Award. In 2012, he received the IEEE Canada McNaughton Gold Medal and the Queen Elizabeth II Diamond Jubilee Medal. In 2013, he received the IEEE MTT-S Microwave Career Award. In 2014, he received McMaster Universitys Faculty of Engineering Research Achievement Award. In 2016 he was honored by appointment to Officer of the Order of Canada; and in 2018, Professional Engineers Ontario awarded him their Gold Medal. Dr. Bandler pioneered space mapping in 1993. 